\title{
How Fast can a Distributed Atomic Read be? ${ }^{1}$
}

\author{
PARTHA DUTTA, RACHID GUERRAOUI, RON R. LEVY and MARKO VUKOLIC \\ School of Computer \& Communication Sciences, EPFL, Lausanne, Switzerland
}

We study efficient and robust implementations of an atomic read-write data structure over an asynchronous distributed message-passing system made of reader and writer processes, as well as failure prone server processes implementing the data structure. We determine the exact conditions under which every read operation involves one-round of communication with the servers. These conditions relate the number of readers to the tolerated number of faulty servers, in a general model with crash and arbitrary failures.

Categories and Subject Descriptors: C.2.1 [Computer-Communication Networks]: Network Architecture and Design-distributed networks; C.2.4 [Computer-Communication Networks]: Distributed Systems; D.4.1 [Operating Systems]: Process Management-concurrency, multiprocessing/multiprogramming, synchronization; F.1.1 [Computation by Abstract Devises]: Models of Computation-relations among models

General Terms: Algorithms, Theory, Reliability

Additional Key Words and Phrases: atomic registers, shared-memory emulation, time-complexity

\section{INTRODUCTION}

Assigning a value to a variable and fetching a value from a variable are probably the most common instructions of any program. When several programs cooperate to achieve a common task, it is natural to provide them with means to perform those instructions through shared variables.

The atomic read-write data structure allows concurrent processes, each possibly running a different program, to share information through a common variable, as if they were accessing this variable in a sequential manner. This abstraction, usually called an atomic register [Lamport 1986] or simply a register, is fundamental in distributed computing and is at the heart of a large number of distributed algorithms [Herlihy 1991; Attiya and Welch 1998].

We study distributed implementations of this abstraction in a message passing system with no physical shared memory: a set of server processes provide the illusion to a set of reader and writer processes (clients) that the abstraction is a physical memory accessible to the clients.

We consider robust [Attiya et al. 1995], or wait-free [Herlihy 1991], implementations of this data structure where any read or write invocation by some client process eventually returns, independently of the operational status of other clients: all of them might have stopped their computation. The data structure itself is replicated over several servers to tolerate the failure of some of these servers.

Ensuring both atomicity and robustness is not trivial. Informally, atomicity requires that, even though each read or write operation may take an arbitrary period of time to complete, they appear to execute at some instant during their respective period of execution [Lamport 1986]. This requires ordering operations in a way that respects their physical order as well as the expected sequential specification of

\footnotetext{
${ }^{1}$ Elements of this paper appeared in a preliminary form in a paper with the same title in the proceedings of the ACM Symposium on Principles of Distributed Computing, 2004.
} 
a read-write data structure: namely, a read should return the last written value.

To illustrate how an implementation can be robust yet achieve atomicity and motivate our quest for efficient implementations, consider the classical implementation from [Attiya et al. 1995] in the case of a single-writer multi-reader case, also called an SWMR register [Lamport 1986]. In [Attiya et al. 1995], readers and servers are the same set, the writer is one of the servers, and a minority of processes may fail by crashing, i.e., halting all their activities.

This implementation maintains the required order among operations by associating timestamps with every written value. To write some value $v$, the writer increments its local timestamp, and sends $v$ with the new timestamp ts to all servers. Every server, on receiving such a message, stores $v$ and $t s$ and then sends an acknowledgment (an ack) to the writer. On receiving acks from a majority, the writer terminates the write. In a read operation, the reader first gathers value and timestamp pairs from a majority of servers, and selects the value $v$ with the largest timestamp $t s$. Then the reader sends $v$ and $t s$ to all servers, and returns $v$ on receiving acks from a majority of processes. Unlike the writer ${ }^{2}$, a reader does not know the latest timestamp in the system, and hence, needs to spend one communication round-trip to discover the latest value, and then another round-trip to propagate the value to a majority of servers. The second round-trip is "required" because the latest value learned in the first round-trip might be present at only a minority of servers. In a sense, every read includes, in its second communication round-trip, a "write phase", with the input parameter being the value selected in the first round-trip.

It is easy to see how to reduce the time-complexity of a read by using a simple decentralization combined with a max-min technique. First, the reader sends messages to all servers. Every server, on receiving such a message, broadcasts its timestamp to all servers. On receiving timestamps from a majority of servers, every server selects the maximum timestamp, adopts the timestamp and its associated value, and sends the pair to the reader. On receiving such messages from a majority of servers, the reader returns the value with the minimum timestamp. To see why this ensures atomicity, observe that, when a write completes, its timestamp, say $t s$, is stored at a majority of servers. In any subsequent read, every server sees a timestamp that is higher than $t s$, before the server sends the message to the reader. Hence, the read returns a value that is not older than the written value. On the other hand, if a read returns a value with timestamp $t s$, then a majority of servers have a timestamp not lower than $t s$, and no subsequent read returns an older value.

But can we do better? Is there a fast implementation where none of the operations (read or write) require more than one communication round-trip? This would clearly be optimal in terms of time-complexity.

With a single reader, it is easy to modify the algorithm of [Attiya et al. 1995] such that the read takes only one round-trip: the read can return the latest value learned from the servers in the first round trip, provided it is not older than the

\footnotetext{
${ }^{2}$ Given the single-writer setting, and since only the writer introduces new timestamps in the system, the writer always knows the latest timestamp. Thus, on invoking a write operation, the writer just needs to increment its own timestamp to get a timestamp that is higher than any existing timestamp in the system.
} 
value returned in the previous read. Otherwise, the reader returns the same value as in the previous read. Since there is only one reader, this clearly orders the reads in the desired fashion and ensures atomicity. To illustrate this case, suppose the writer writes $v$ with timestamp 7, and the write message is received only by one server $s$. (The write is incomplete.) The first reader gets information from a majority of servers that includes $s$. The read must return $v$ because the reader does not know whether the write of $v$ is complete or not, and this reader has to return the value of the last preceding write.

Consider now the situation with another reader. The second reader invokes a read, queries a majority of servers, and misses $s$. Clearly, the second read returns a value with a timestamp lower than 7 , violating atomicity: the second read returns an older value than the preceding read.

At first glance, it seems impossible to have a fast implementation with two readers when $t<S / 2$. But what if we tolerate fewer faulty servers?

We show in this paper that, interestingly, the existence of a fast SWMR implementation depends on the maximum number $R$ of readers. We consider a general model where $t$ among the set $S$ of server processes on which the data structure is implemented can fail by crashing, or even deviate arbitrarily from their algorithm and be malicious: we denote by $b \leq t$ the number of malicious server failures.

We show that there is a fast implementation of a SWMR register if and only if the number of readers $R$ is less than $\frac{S+b}{t+b}-2$.

For simplicity of presentation, we first prove our result for the crash-stop case. $\left(R<\frac{S}{t}-2\right)$, and later generalize it to arbitrary failures (i.e., assuming $\left.b \neq 0\right)$. We first give an algorithm (i.e., a fast implementation) and then a lower bound (i.e., we prove the implementation is optimal).

- To get an intuition of our fast implementation, consider the algorithm sketched above [Attiya et al. 1995] and the following observation: if a reader sees the latest timestamp $t s$ at $x$ servers, then any subsequent reader sees $t s$ or a higher timestamp at $x-t$ servers; this is because, in a fast implementation, the first reader does not propagate $t s$, and the second reader might miss $t$ servers seen by the first reader. A generalization of this observation helps determine when some reader can safely return the value associated with the latest timestamp. This is not entirely trivial because the atomicity of a value can not be simply deduced from the number of servers that has seen the value. To determine whether a value is safe to return, we have every server maintain, besides the latest value, the set of readers to which the server has sent that value.

- Given $S$ and $t$, we prove by contradiction that there is no fast implementation with $R \geq \frac{S}{t}-2$. Given a fast implementation with $R \geq \frac{S}{t}-2$, we consider a partial run which contains a write(1) that misses $t$ servers, and we append it with a read that misses $t$ other servers. Then we delete all the steps in the partial run that are not "visible" to the reader (basically, the steps of the $t$ servers that the read missed). By atomicity, the read returns 1 in the resulting partial run. Now we iteratively append reads by distinct readers, and delete the steps in the partial run that are not visible to the last reader, until we exhaust all the readers. To ensure atomicity, the last read of each partial run returns 1 . In the final partial run (obtained after exhausting all readers) the steps of write(1) are 
almost deleted. We modify this partial run to construct several additional partial runs, one of which violates atomicity.

- Our algorithm and lower bound are then extended, after a careful analysis of the impact of malicious servers, to the more general situation with arbitrary failures. We assume that, out of the $t$ servers than can fail, up to $b \leq t$ processes can be malicious and we show that a fast implementation is possible if and only if the number of readers is less than $\frac{S+b}{t+b}-2$.

- To complete the picture, we prove that it is impossible to have a one-round read algorithm with multiple writers [Lynch and Shvartsman 1997] (MWMR atomic register) even if only one server can fail and it can only do so by crashing.

The paper is organized as follows. Section 2 gives the system model and defines fast atomic implementations. We present a fast implementation assuming $R<\frac{S}{t}-2$ in Section 4. We prove a tight bound for $R$ in Section 5. Section 6 extends the previous results to the arbitrary failure model. Section 7 considers the multi-writer case. Section 8 discusses the impact of our results on the folklore theorem that every "atomic read must write". Section 9 summarizes the main results of the paper.

\section{MODEL AND DEFINITIONS}

\subsection{Basics}

The distributed system we consider consists of three disjoint sets of processes: a set servers of size $S$ containing processes $\left\{s_{1}, \ldots, s_{S}\right\}$, a set writer containing a single process $\{w\},{ }^{3}$ and a set readers of size $R$ containing processes $\left\{r_{1}, \ldots, r_{R}\right\}$. Every pair of processes communicate by message-passing using a bi-directional reliable communication channel.

A distributed algorithm $A$ is a collection of automata, where $A_{p}$ is the automata assigned to process $p$. Computation proceeds in steps of $A$. A step of algorithm $A$ is denoted by a pair of process id and a set of messages received in that step $<p, M>(M$ might be $\emptyset)$. A run is an infinite sequence of steps of $A$. A partial run is a finite prefix of some run. A (partial) run $r$ extends some partial run $p r$ if $p r$ is a prefix of $r$. At the end of a partial run, all messages that are sent but not yet received are said to be in transit. In any given run, any number of readers, the writer, and $t$ out of $S$ servers may crash.

\subsection{Details of the System Model}

The state of communication channels is viewed as a set of messages mset containing messages that are sent but not yet received. We assume that every message has two tags which identify the sender and the receiver of the message. A distributed algorithm $A$ is a collection of automata, where $A_{p}$ is the automata assigned to process $p$. Computation proceeds in steps of $A$. A step of $A$ is denoted by a pair of process id and message set $\langle p, M>(M$ might be $\emptyset)$. In step $\langle p, M>$, process $p$ atomically does the following: (1) remove the messages in $M$ from mset, (2) apply $M$ and its current state $s t_{p}$ to $A_{p}$, which outputs a new state $s t_{p}^{\prime}$ and a set of

\footnotetext{
${ }^{3}$ We discuss the multi-writer case in Section 7 .
} 
messages to be sent, and then (3) $p$ adopts $s t_{p}^{\prime}$ as its new state and puts the output messages in mset.

Given any algorithm $A$, a run of $A$ is an infinite sequence of steps of $A$ such that the following properties hold for each process $p$ : (1) initially, mset $=\emptyset,(2)$ the current state in the first step of $p$ is a special state Init, (3) for each step $<p, M>$, and for every message $m \in M, p$ is the receiver of $m$ and $m s e t$ contains $m$ immediately before the step $\langle p, M\rangle$ is taken, and (4) if there is a step that puts a message $m$ in mset such that $p$ is the receiver of $m$ and $p$ takes an infinite number of steps, then there is a subsequent step $<p, M>$ such that $m \in M$.

A partial run is a finite prefix of some run. We say that a process is correct in a run if it takes an infinite number of steps in that run. Otherwise the process is faulty. In a run of our model, any number of readers or the writer may be faulty, and at most $t \leq S$ servers might be faulty. We say that a (faulty) process $p$ crashes at step $s p$ in a run, if $s p$ is the last step of $p$ in that run.

For presentation simplicity, we do not explicitly model the initial state of a process, nor the invocations and responses of operations. We assume that the algorithm $A$ initializes the processes, and schedules invocation/response of operations (i.e., $A$ modifies the states of the processes accordingly). However, we say that $p$ invokes op at step $s p$, if $A$ modifies the state of a process $p$ in step $s p$ so as to invoke an operation (and similarly for response).

\section{ATOMICITY}

A history of a partial run is a sequence of invocation and response steps of read or write operations in the same order as they appear in the partial run. An incomplete invocation step in a history is an invocation step without a matching response step in that history. We say that a history $H 1$ completes history $H 2$ if $H 1$ can be obtained through the following modification of $\mathrm{H} 2$ : for each incomplete invocation step $s p$ in $H 2$, either $s p$ is removed from $H 2$, or any valid matching response for that invocation is appended to the end of $H 2$.

\subsection{Atomic Register}

A sequential register is a data structure accessed by a single process. It provides two operations: write $(v)$, which stores $v$ in the register, and read(), which returns the last value stored. An atomic register is a distributed data structure that may be concurrently accessed by multiple processes and yet provides an "illusion" of a sequential register to the accessing processes.

We refer the readers to [Lamport 1986; Lynch 1996; Herlihy 1991; Herlihy and Wing 1990] for a formal definition of an atomic register, and we simply recall below what is required to state and prove our results.

We assume that each process invokes at most one invocation at a time (i.e., does not invoke the next operation until it receives the response for the current operation). Only readers invoke reads on the register and only the writer invokes writes on the register. We further assume that the initial value of a register is a special value $\perp$, which is not a valid input value for a write. In any run, we say that an operation op 1 precedes operation op 2 (or op 2 succeeds op 1 ) if the response step of op 1 precedes the invocation step of op 2 in that run. If neither op 1 nor op 2 precedes the other, the operations are said to be concurrent. We say that an operation is 


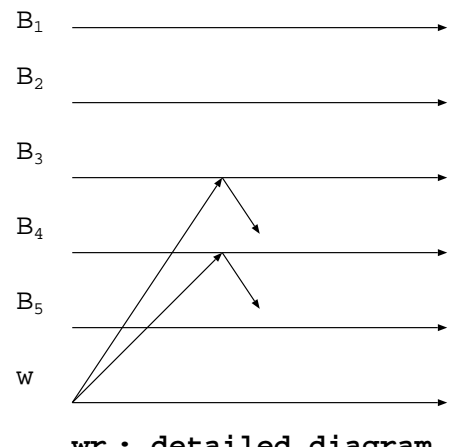

$\mathrm{wr}_{3}$ : detailed diagram

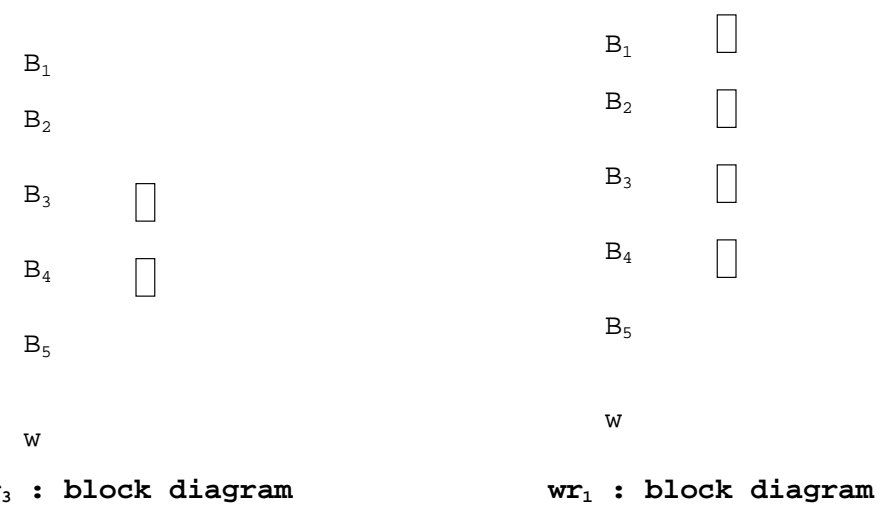

Fig. 1. Partial writes: $w r_{i}$

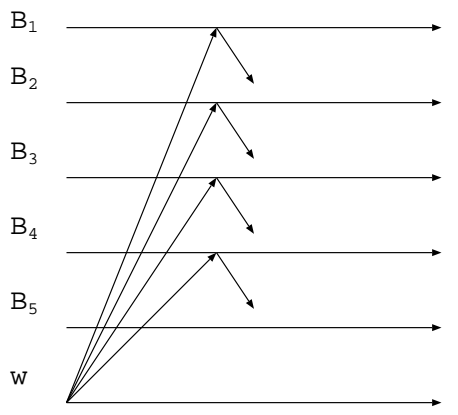

wr $r_{1}$ : detailed diagram

complete in a (partial) run if the run contains a response step for that operation.

An algorithm implements a register if every run of the algorithm satisfies termination and atomicity properties. Termination states that if a process invokes an operation, then eventually, unless that process crashes, the operation completes (even if all other client processes have crashed). Here we give a definition of atomicity for the single-writer registers.

In the single-writer setting, the writes in a run have a natural ordering which corresponds to their physical order. Denote by $w r_{k}$ the $k^{\text {th }}$ write in a run $(k \geq 1)$, and by $v a l_{k}$ the value written by the $k^{t h}$ write. Let $v a l_{0}=\perp$. We say that a partial run satisfies atomicity if the following properties hold: (1) if a read returns $x$ then there is $k$ such that $v_{a l}=x,(2)$ if a read $r d$ is complete and it succeeds some write $w r_{k}(k \geq 1)$, then $r d$ returns $v_{a l}$ such that $l \geq k$, (3) if a read $r d$ returns $v_{a l}(k \geq 1)$, then $w r_{k}$ either precedes $r d$ or is concurrent to $r d$, and (4) if some read $r d 1$ returns $v_{a l}(k \geq 0)$ and a read $r d 2$ that succeeds $r d 1$ returns $v a l_{l}$, then $l \geq k$.

\subsection{Fast Implementations}

Basically, we say that a read or a write operation is fast if it completes in one communication round-trip. In other words, in a fast read:

(1) The reader sends messages to a subset of processes in the system (possibly all 
processes).

(2) Processes on receiving such a message reply to the reader before receiving any other messages. More precisely, any process $p$ on receiving a message $m$ in step $s p 1=<p, M>(m \in M)$, where $m$ is sent by a reader on invoking a read, replies to $m$ either in step $s p 1$ itself, or in a subsequent step $s p 2$, such that $p$ does not receive any message in any step between $s p 1$ and $s p 2$ (including $s p 2$ ). Intuitively, this requirement forbids the processes to wait for some other message before replying to $m$.

(3) the reader on receiving a sufficient number of such replies returns from the read.

Recall that implementations need to tolerate the crash of the writer, any reader, and up to $t$ servers. Hence, in order to ensure termination, the reader cannot wait for replies from any other reader, or writer, or more than $S-t$ servers. We similarly say that a write operation is fast if it completes in one round-trip.

We say that an implementation has fast reads (or writes) if every complete read (resp. complete write) operation in every run is fast. A fast implementation is an implementation in which both reads and writes are fast. For an implementation that has fast reads, we can say without ambiguity that the messages sent by a reader, on invoking a read, are of type READ, and the messages sent by a process to the reader, on receiving a READ message, of type READACK. Similarly, we define WRITE and WRITEACK messages for fast writes.

\section{A FAST IMPLEMENTATION}

We describe in this section a fast implementation assuming $R<\frac{S}{t}-2$ (the pseudo code of the implementation is given in Figure 2). For simplicity of presentation, we assume that the writer writes timestamps, and the readers read back timestamps. We ignore the value associated with the timestamp for now. Later we explain how to trivially modify our algorithm such that the writer and the readers associate some value with a timestamp.

The procedure for write is similar to that in [Attiya et al. 1995]. On invoking a write, the writer increments its timestamp and sends a WRITE message with the timestamp to all servers. Servers on receiving the message store the timestamp, and send WRITEACK messages back to the writer. The writer returns OK once it has received WRITEACK messages from $S-t$ servers.

Implementing a fast read is more involved. Recall that, to maintain atomicity, a read needs to return a timestamp that is not lower than the timestamp of the last completed write, and has to guarantee that no subsequent read returns a lower timestamp.

Our read procedure collects timestamps from $S-t$ servers (by sending READ messages and receiving READACK messages from the servers), and selects the highest timestamp, denoted by $\max T S$ in Figure 2. Then the reader checks if $\max T S$ has been seen by a "sufficient" number of servers and readers. If so, the read returns $\max T S$, else it returns $\max T S-1$. The heart of the algorithm is the predicate for checking whether the latest value has been seen by a sufficient number of processes:

(1) The predicate is true whenever the write with timestamp maxTS precedes the current read. 
1: at the writer $w$

procedure initialization:

ts $\leftarrow 1$, rCounter $\leftarrow 0$

procedure write $(v)$

send(WRITE, ts, rCounter) to all servers

wait until receive(WRITEACK, $t s, *, r$ Counter) from $S-t$ servers

$t s \leftarrow t s+1$

$\operatorname{return}(\mathrm{OK})$

9: at each reader $r_{i}$

procedure initialization:

$t s \leftarrow 0 ; r$ Counter $\leftarrow 0 ; \max T S \leftarrow 0$

procedure read()

$r$ Counter $\leftarrow$ Counter $+1 ;$ ts $\leftarrow \max T S$

send(READ, ts, rCounter) to all servers

wait until receive(READACK, $*, *$, rCounter) from $S-t$ servers

$r c v M s g \leftarrow\left\{m \mid r_{i}\right.$ received (READACK, $*, *, r$ Counter) $\}$

$\max T S \leftarrow \operatorname{Maximum}\left\{t s^{\prime} \mid\left(\mathrm{READACK}, t s^{\prime}, *, r\right.\right.$ Counter $) \in$ rcvMsg $\}$

$\max T S m s g \leftarrow\{m \mid m . t s=\max T S$ and $m \in \operatorname{rcv} M s g\}$

if there is $a \in[1, R+1]$ and there is MS $\subseteq$ maxTSmsg s.t., $(|M S| \geq S-a t)$ and

$\left(\left|\cap_{m \in M S} m \cdot s e e n\right| \geq a\right)$ then

$\operatorname{return}(\max T S)$

else

$\operatorname{return}(\max T S-1)$

23: at each server $p_{i}$

4: procedure initialization:

ts $\leftarrow 0 ;$ seen $\leftarrow \emptyset ;$ counter $[0 \ldots R] \leftarrow[0 \ldots 0]$

26: upon receive(msgType, $t s^{\prime}$, rCounter $\left.^{\prime}\right)$ from $q \in\left\{w, r_{1}, \ldots, r_{R}\right\}$ and Counter $^{\prime} \geq$ counter $[\operatorname{pid}(q)]$ do

if $t s^{\prime}>t s$ then

$t s \leftarrow t s^{\prime} ;$ seen $\leftarrow\{q\}$

else

seen $\leftarrow$ seen $\cup\{q\}$

counter $[\operatorname{pid}(q)] \leftarrow$ Counter ${ }^{\prime}$

if $m s g T y p e=\mathrm{READ}$ then

send(READACK, ts, seen, rCounter ${ }^{\prime}$ ) to $q$

else

send(WRITEACK, ts, seen, rCounter ${ }^{\prime}$ ) to $q$

Fig. 2. Fast SWMR atomic register implementation with $R<\frac{S}{t}-2$

(2) If there is no write with a timestamp higher than $\max T S$, then if the predicate is true for the current read, it is also true for all subsequent reads.

In order to construct such a predicate however, the servers need to record more information than just the latest timestamp, as we explain below.

Consider the case of a write with timestamp ts that is followed by a read:

- In the first partial run $p r_{1}$, the write completes by writing $t s$ at $S-t$ servers, say the set of servers be $S_{1}$. Subsequently, a reader reads from a set $S_{2}$ (of $S-t$ servers) that overlaps at $S-2 t$ servers with $S_{1}$, i.e., misses $t$ servers in $S_{1}$. By atomicity, the read returns $t s$.

- In the second partial run $p r_{2}$, the write is incomplete and the writer writes $t s$ 
only to $S-2 t$ servers in $S 1 \cap S 2$. A subsequent reader that reads from $S_{2}$ cannot distinguish $p r_{1}$ from $p r_{2}$, and returns $t s$.

If we extend each partial run with another read by a distinct reader that misses $t$ servers from $S 1 \cap S 2$, it is easy to see that the new read has to return $t s$, even if it sees $t s$ at $S-3 t$ servers that have already replied to both the write and the first read. Thus, we see that any reasonable predicate for fast reads must depend on the number of servers, as well as the number of readers, that have seen the most recent timestamp. Since any number of readers might crash, a reader cannot wait for the replies from other readers, but rather indirectly collect information about other readers from the servers.

Generalizing the above argument gives us the desired predicate. Along with the latest timestamp $t s$, every server maintains the list of readers and writer to which the server has replied after updating its timestamp to $t s$ (including the reader or the writer which updated the timestamp of the server to $t s$ ). This set is denoted by seen in Figure 2. The predicate for the read procedure is as follows: if there is $a \geq 1$ such that the reader receives $\max T S$ in at least $S$-at messages, and there are at least $a$ processes that are in the list seen of each of these $S-a t$ messages, then the predicate is true.

In addition, every reader $r_{i}$ maintains a variable rCounter that counts the number of reads of $r_{i}$. The servers maintain an array, counter, such that counter $[i]$ contains the latest value of $r$ Counter that the server has received from $r_{i}$. In the algorithm, $\operatorname{pid}(q)$ is a function that maps the writer $w$ to 0 , and every reader $r_{i}$ to $i$. This helps distinguish READ and READACK messages from different reads of the same reader. At the writer, the variable $r$ Counter is always 0 ; the messages from different writes are distinguished by their respective timestamps.

This completes the brief description of the register implementation. We now describe how to modify the algorithm so as to associate values with timestamps. In the modified algorithm, in each write, the writer attaches two tags with the timestamp, containing the current value to be written and the value of the immediately preceding write. If the reader returns $\max T S$ in the original algorithm, then it returns the current value attached to $\max T S$ in the modified algorithm. If the reader returns $\max T S-1$ in the original algorithm, it returns the other tag attached to $\max T S$ in the modified algorithm.

We now prove the correctness of the fast implementation in Figure 2. We do not assume that the lines in Figure 2 are atomic: processes may crash in the middle of a line or in between two lines. In particular, while sending messages to a set of processes, the sending process may crash after sending messages to an arbitrary subset. We assume that, if a process receives an incomplete message, the process can detect that the message is incomplete, and ignores such a message.

It is obvious that read and write procedures complete in one round-trip. To show atomicity, we recall that the write procedure directly writes the timestamp. Thus the conditions in Section 3.1 reduce to the following:

(1) If a read returns, it returns a non-negative integer.

(2) If a read $r d$ is complete and it succeeds some write $(k)$, then $r d$ returns $l$ such that $l \geq k$. 
(3) If a read $r d$ returns $k(k \geq 1)$, then write $(k)$ either precedes $r d$ or is concurrent to $r d$.

(4) If some read $r d 1$ returns $k(k \geq 0)$ and a read $r d 2$ that succeeds $r d 1$ returns $l$, then $l \geq k$.

The proofs of the first and the third conditions are trivial. Below, we show the other two. In the proofs we refer to the global clock; however processes do not access this global clock.

LEMMA 1. If a server sets ts to $x$ at time $T$, then the server never sets ts to a value that is lower than $x$ after time $T$.

PRoOF: obvious from line 27.

LEMMA 2. If a read() sends READ messages with $t s=x$, then the read does not return a value smaller than $x$.

PRoOF: suppose read $r d$ by $r_{i}$ sends a READ message with $t s=x$. From line 27 , every READACK message received by $r d$ is with $t s \geq x$. Let $z$ be the maximum timestamp received by $r d$ (i.e., $\max T S$ computed in line 17). Notice that $r d$ returns either $z$ or $z-1$. There are the following two cases to consider. (1) If $z>x$, then clearly, the return value is not smaller than $x$. (2) If $z=x$. then every READACK message received by $r d$ has $t s=x$ and has $p_{i} \in$ seen. Since $r d$ receives $S-t$ READACK messages, the predicate in line 19 of $r d$ holds with $a=1$. Hence, $r d$ returns $x$.

LEMMA 3. If a read $r d$ is complete and it succeeds some write $(k)$, then $r d$ returns $l$ such that $l \geq k$.

PROOF: suppose that write $w r$ (by $w$ ) writes $k$ and precedes read $r d$ (by reader $r_{j}$ ). Let $S 1$ be the set of $S-t$ servers from which $w r$ received WRITEACK messages in line 6 , and let $S 2$ be the set of $S-t$ servers from which $r d$ received READACK messages in line 15 . Let $S 12=S 1 \cap S 2$. Obviously, $|S 12| \geq S-2 t$. Let $z$ be the maximum timestamp received by $r d$ from servers in $S 2$. Observe that $r d$ returns either $z$ or $z-1$.

When a server in $S 1$ replies to a WRITE message from $w r$, its $t s$ is $k$. The server's $t s$ is not higher than $k$ because, unless the writer receives WRITEACK from all servers in $S 1$, it does not complete write $(k)$, and hence, no timestamp higher than $k$ is present in the system until all servers in $S 1$ reply to write $(k)$. From Lemma 1, servers in $S 1$ (and hence, in $S 12$ ) reply $t s \geq k$ to $r d$ because write $(k)$ precedes $r d$ ). Thus, the highest timestamp received by $r d, z \geq k$. There are the following two cases to consider:

1. $z>k$

Since $r d$ returns either $z$ or $z-1$, it follows that $r d$ does not return a timestamp lower than $k$.

2. $z=k$

We know that every server in $S 12$ replies to $r d$ with $t s \geq k$, and $z=k$ is the 
maximum timestamp received by $r d$ from servers in $S 2 \supseteq S 12$. Thus every server in $S 12$ replies $t s=k$ to $r d$. Let $M S$ be the set of READACK messages sent by servers in $S 12$ to $r d$. Since every server in $S 12$ replies $t s=k$ to $w r$ before sending $t s=k$ to $r d$, for every message $m$ in $M S, w \in m$.seen. Furthermore, from line $30, r_{j} \in$ m.seen. Thus, $\left\{w, r_{j}\right\} \subseteq \cap_{m \in M S}$ m.seen. As $|S 12| \geq S-2 t$, in $r d$, the predicate in line 19 holds with $a=2$. Consequently, $r d$ returns $z=k$.

Lemma 4. If some read $r d 1$ returns $x(x \geq 0)$ and a read $r d 2$ that succeeds $r d 1$

returns $y$, then $y \geq x$.

Proof: suppose that read $r d 1$ by process $r_{j}$ returns $x$, read $r d 2$ by process $r_{k}$ returns $z$, and $r d 1$ precedes $r d 2$. Suppose $r_{j}=r_{k}$. Then, in the read immediately after $r d 1, r_{j}$ sends a READ message with $t s \geq x$, and hence, from Lemma 2 , the read returns a value greater than or equal to $x$. Using Lemma 2 and a simple induction, we can derive that any read by $r_{j}$ which follows $r d 1$ (including $r d 2$ ) returns $t s \geq x$. So in the rest of the proof we assume that $r_{j} \neq r_{k}$.

Let $S 1$ and $S 2$ be the set of servers (of size $S-t$ ) from which reads $r d 1$ and $r d 2$, respectively, receive $S-t$ READACK messages in line 15 . Let $T S 1$ be the highest timestamp received by $r d 1$ from processes in $S 1$ (i.e., the $\max T S$ evaluated in line 17 of $r d 1$ ). Similarly, let $T S 2$ be the highest timestamp received by $r d 2$ from the processes in $S 2$. There are the following two cases to consider:

$\langle 1\rangle 1$. the predicate in line 17 does not hold in $r d 1$.

It follows that $x=T S 1-1$. Thus some servers have sent $t s=T S 1=x+1$ to $r d 1$, and hence, write $(x+1)$ has started before $r d 1$ is completed. Thus write $(x)$ has completed before $r d 1$ is completed. Since $r d 1$ precedes $r d 2$, it follows that write $(x)$ precedes $r d 2$. From Lemma $3, r d 2$ returns $y \geq x$.

$\langle 1\rangle 2$. the predicate in line 17 holds in $r d 1$.

It follows that $x=T S 1$, and there is some $a \in[1, R+1]$ such that there is a set $M S$ consisting of at least $S-a t$ messages received by $r d 1$ with $t s=x$ and $\left|\cap_{m \in M S} m . s e e n\right| \geq a$. Let $S 12 \subseteq S 1$ be the set of servers which sent the messages that are in $M S$. Since $a \in[1, R+1]$ and $t<S /(R+2),|S 12|=|M S|=S-a t>t$. There are the following two cases to consider:

$\langle 2\rangle$ 1. $y=T S 2$

$y=T S 2$. Since, $|S 12|>t$ and $|S 2|=S-t$, there is a server $p_{i} \in S 2 \cap S 12$.

Since $r d 1$ precedes $r d 2, p_{i}$ first replies $t s=x$ to $r d 1$ then replies to $r d 2$. From

Lemma 1, it follows that $p_{i}$ replies to $r d 2$ with $t s \geq x$. Thus the highest $t s$ in $S 2$ (i.e., $T S 2=y$ ) is greater than or equal to $x$.

$\langle 2\rangle 2 . y=T S 2-1$

There are the following two subcases to consider:

$\langle 3\rangle 1 . y+1 \neq x$

As in case $\langle 2\rangle 1$, we can show that there is a server $p_{i} \in S 2 \cap S 12$, and $p_{i}$ replies to $r d 2$ with $t s \geq x$. Thus the highest $t s$ in $S 2$ (i.e., $T S=y+1$ ) is greater than or equal to $x$. Since $y+1 \neq x$, it follows that $y+1>x$, and hence, $y \geq x$.

$\langle 3\rangle 2 . y+1=x$

Consider the set of servers $S 2 \cap S 12$. As $|S 12|=S-a t$ and $|S 2|=S-t$, so $|S 2 \cap S 12| \geq S-(a+1) t \geq 1$. Since $r d 1$ precedes $r d 2$ and processes in $S 12$ 
replies $t s=x$ to $r d 1$, processes in $S 2 \cap S 12$ reply to $r d 2$ with $t s \geq x$. Since $y+1$ is the maximum $t s$ in $S 2$, every process in $S 2 \cap S 12$ replies to $r d 2$ with $t s=x=y+1$. There are the following two cases to consider:

$\langle 4\rangle 1 . a \leq R$

Then $|S 2 \cap S 12| \geq S-(a+1) t>t$. Let $M S 1$ be the set of READACK messages from processes in $S 2 \cap S 12$ to $r d 1$. From the definition of $M S 1$ and $M S, M S 1 \subseteq M S .{ }^{4}$ Thus, $\cap_{m \in M S 1}$ m.seen $\supseteq \cap_{m \in M S}$ m.seen. Thus, $\mid \cap_{m \in M S 1}$ m.seen $\mid \geq a$. There are two cases to consider:

$\langle 5\rangle$. $r_{k} \notin \cap_{m \in M S 1}$ m.seen

Let $M S 2$ be the set of messages received by $r d 2$ from processes in $S 2 \cap$ $S 12$. For any server $p_{i} \in S 2 \cap S 12$, let $m 1_{i}$ and $m 2_{i}$ be the messages sent by $p_{i}$ in $M S 1$ and $M S 2$ respectively. We know that $m 1_{i}$.ts $=m 2_{i}$.ts $=$ $x$. Since $m 1_{i}$ is sent before $m 2_{i}$ and the $t s$ is the same in both messages, $m 1_{i}$.seen $\subseteq m 2_{i}$.seen. Thus $\cap_{m \in M S 1} m$.seen $\subseteq \cap_{m \in M S 2} m$.seen. Since every process which replies to $r d 2$, first adds $r_{k}$ to its seen set, $r_{k} \in$ $\cap_{m \in M S 2}$ m.seen. Since $r_{k} \notin \cap_{m \in M S 1}$ m.seen, it follows that $\mid \cap_{m \in M S 2}$ m.seen $|\geq| \cap_{m \in M S 1}$ m.seen $\mid+1 \geq a+1$. Since $|S 2 \cap S 12| \geq S-(a+1) t$, the number of message in $M S 2$ is at least $S-(a+1) t$. As $a+1 \leq R+1$, the predicate in line 19 in $r d 2$ holds with $a+1$. Thus, the timestamp returned by $r d 2$ is $x=y+1$, a contradiction.

$\langle 5\rangle 2 . r_{k} \in \cap_{m \in M S 1}$ m.seen

Thus each server $p_{i}$ in $S 2 \cap S 12$ has sent at least one READACK message with $t s=x$ to $r_{k}$, before $p_{i}$ sent the $M S 1$ message to $r_{j}$. Since the messages in $M S 1$ are sent before the completion of $r d 1$ (and hence, before the invocation of $r d 2$ ), $r_{k}$ has invoked at least one read before $r d 2$. Let $r d 2 a$ be the last read of $r_{k}$ which precedes $r d 2$. Since $|S 2 \cap S 12| \geq$ $S-(a+1) t>t$, there is at least one process $p_{i}$ in $S 2 \cap S 12$ whose READACK message is received by $r d 2 a$, say message $m$. Now consider the last READACK message sent by $p_{i}$ to $r_{k}$ before $r d 2$ is invoked, say message $m^{\prime}$. Since we know that $p_{i}$ sent a READACK message with $t s=x$ to $r_{k}$ before sending a $M S 1$ message (which was in turn sent before $r d 2$ was invoked), from Lemma 1 it follows that $m^{\prime}$ was sent with $t s \geq x$. We now claim that $m=m^{\prime}$. By definition of $m^{\prime}$, either $m=m^{\prime}$ or $m^{\prime}$ is sent after $m$. Observe that $p_{i}$ checks counter $[k]$ before replying to $r_{k}$. Thus, once $m$ is sent by $p_{i}$, counter $[k]$ at $p_{i}$ is set such that $p_{i}$ can only reply to those message of $r_{k}$ which are sent from $r d 2 a$ or a subsequent read of $r_{k}$. Thus, if $m^{\prime}$ is sent after $m$, then $m^{\prime}$ is sent in response to $r d 2 a$, or $r d 2$, or a subsequent read of $r_{k}$. This contradicts the assumption that $p_{i}$ replies only once to $r d 2 a$ (because channels do not duplicate messages) and $m^{\prime}$ is sent before $r d 2$ is invoked. Thus $r d 2 a$ receives $m=m^{\prime}$. We have already shown that $m^{\prime}$ is sent with $t s \geq x$. Hence the highest $t s$ received by $r d 2 a$ is greater than or equal to $x$. It follows that $r d 2$ sends READ messages with $t s \geq x$. From Lemma $2, r d 2$ returns a timestamp greater than or equal to $x$. As $x=y+1, r d 2$ does

$\overline{{ }^{4} \text { See case }\langle 1\rangle} 2$ for the definition of $M S$. 
not return $y$, a contradiction.

$\langle 4\rangle 2 . a=R+1$

Since $\left|\left\{w, r_{1}, \ldots, r_{R}\right\}\right|=R+1$ and $\mid \cap_{m \in M S}$ m.seen $\mid \geq a=R+1$, we have $r_{k} \in \cap_{m \in M S}$ m.seen. Observe that $|S 12| \geq S-a t>t$. (Recall that $S 12$ is the set of processes which sent the messages that are in $M S$.) Substituting $M S 1$ by $M S$, and $S 2 \cap S 12$ by $S 12$, in the argument for the previous case (case $5\langle 2\rangle$ ), we can show that $r d 2$ returns a value greater than or equal to $x$, a contradiction.

\section{LOWER BOUND}

The following proposition states that the resilience required by our fast implementation is indeed necessary.

Proposition 5. Let $t \geq 1$ and $R \geq 2$. If $R \geq \frac{S}{t}-2$, then there is no fast atomic register implementation.

Preliminaries. Recall first that $w$ denotes the writer, $r_{i}$ for $1 \leq i \leq R$ denote the readers, and $s_{i}$ for $1 \leq i \leq S$ denote the servers. Suppose by contradiction that $R \geq \frac{S}{t}-2$ and there is a fast implementation $I$ of an atomic register. Given that $t \geq S /(R+2)$, we can partition the set of servers into $R+2$ subsets (which we call blocks $)$, denoted by $B_{i}(1 \leq i \leq R+2)$, each of size less than or equal to $t .^{5}$

Since the writer, any number of readers, and up to $t$ servers might crash in our model, the invoking process can only wait for reply messages from $S-t$ servers. Given that we assume a fast implementation, on receiving a READ (or a WRITE) message, the servers cannot wait for messages from other processes, before replying to the READ (or the WRITE) message. We can thus construct partial runs of a fast implementation such that only READ (or WRITE) messages from the invoking processes to the servers, and the replies from servers to the invoking processes, are delivered in those partial runs. All other messages remain in transit. In particular, no server receives any message from other servers, and no invoking process receives any message from other invoking processes. In our proof, we only construct such partial runs.

We say that an incomplete invocation inv skips a set of blocks $B S$ in a partial run, where $B S \subseteq\left\{B_{1}, \ldots, B_{R+2}\right\}$, if (1) no server in any block $B_{i} \in B S$ receives any READ or WRITE message from inv in that partial run, (2) all other servers receive the READ or the WRITE message from inv and reply to that message, and (3) all these reply messages are in transit. We say that a complete invocation inv skips a block $B_{i}$ in a partial run, if (1) no server in $B_{i}$ receives any READ or WRITE message from inv in that partial run, (2) all servers that are not in $B_{i}$ receive the READ or WRITE message from inv and reply to that message, and (3) the invoking process receives all these reply messages and returns from the invocation.

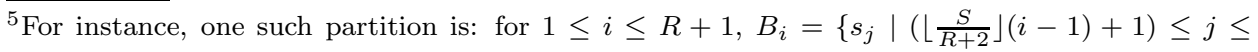
$\left.\left(\left\lfloor\frac{S}{R+2}\right\rfloor i\right)\right\}$, and $B_{R+2}=\left\{s_{j} \mid\left(\left\lfloor\frac{S}{R+2}\right\rfloor(R+1)\right) \leq j \leq S\right\}$. However, if $R>S-2$ then the above partitioning is not possible. In that case we consider a system where, the number of readers is $S-2$ and the set readers is $\left\{r_{1}, \ldots, r_{S-2}\right\}$, and show the impossibility. The impossibility still holds if we add more readers to this system (i.e., $R>S-2$ ).
} 


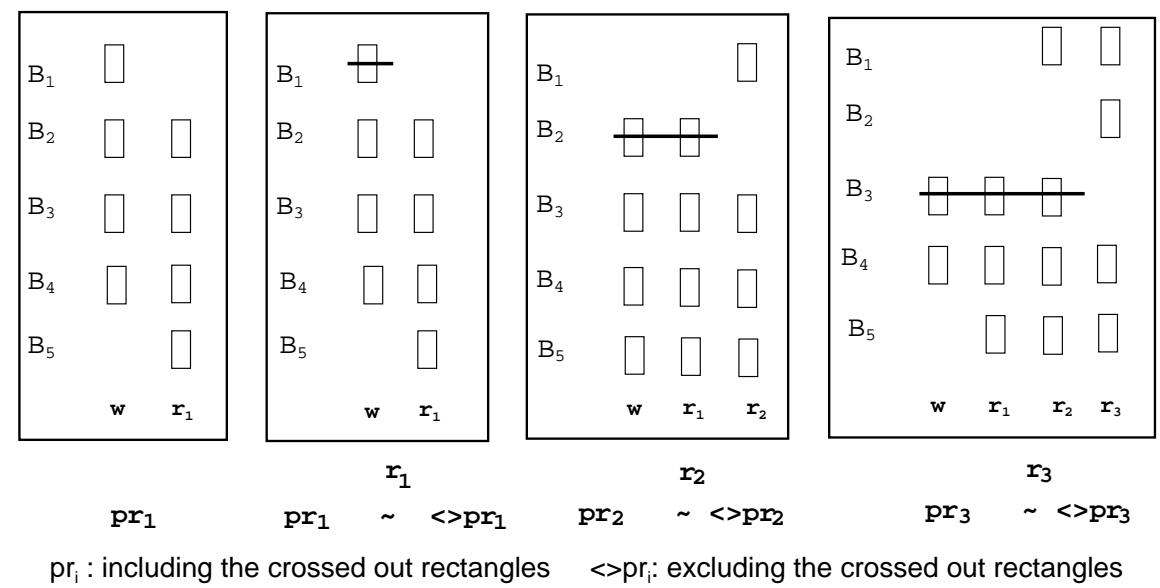

Fig. 3. Partial runs: $p r_{i}$ and $\triangle p r_{i}$

To show a contradiction, we construct a partial run of the fast implementation $I$ that violates atomicity: a partial run in which some read returns 1 and a subsequent read returns an older value, namely, the initial value of the register, $\perp$.

Partial writes. Consider a partial run $w r$ in which $w$ completes write(1) on the register. The invocation skips $B_{R+2}$. We define a series of partial runs each of which can be extended to $w r$. Let $w r_{R+2}$ be the partial run in which $w$ has invoked the write and has sent the WRITE message to all processes, and all WRITE messages are in transit. For $1 \leq i \leq R+1$, we define $w r_{i}$ as the partial run which contains an incomplete write(1) invocation that skips $\left\{B_{R+2}\right\} \cup\left\{B_{j} \mid 1 \leq j \leq i-1\right\}$. We make the following simple observations: (1) for $1 \leq i \leq R, w r_{i}$ and $w r_{i+1}$ differ only at servers in $B_{i}$, (2) $w r$ is an extension of $w r_{1}$, such that, in $w r, w$ receives the replies (that are in transit in $w r_{1}$ ) and returns from the write invocation, and hence, (3) $w r$ and $w r_{1}$ differ only at $w$.

Block diagrams. We illustrate a particular instance of the proof in Figure 3 and Figure 4 , where $R=3$ and the set of servers are partitioned into five blocks, $B_{1}$ to $B_{5}$. We depict an invocation inv through a set of rectangles, (generally) arranged in a single column. In the column corresponding to some invocation $i n v$, we draw a rectangle in the $i^{\text {th }}$ row, if all servers in block $B_{i}$ have received the READ or WRITE message from $i n v$ and have sent reply messages, i.e., we draw a rectangle in the $i^{t h}$ row if $i n v$ does not skip $B_{i}$. (We present a slightly more detailed diagram of the partial writes in Figure 1 in the optional appendix.)

Appending reads. Partial run $p r_{1}$ extends $w r$ by appending a complete read by $r_{1}$ that skips block $B_{1}$. By atomicity, the read returns 1 . Observe that $r_{1}$ cannot distinguish $p r_{1}$ from some partial run $\triangle p r_{1}$, that extends $w r_{2}$ by appending a complete read by $r_{1}$ that skips $B_{1}$. To see why, notice that $w r$ and $w r_{2}$ differ at $w$ and at block $B_{1}$, and $r_{1}$ does not receive any message from these processes in 

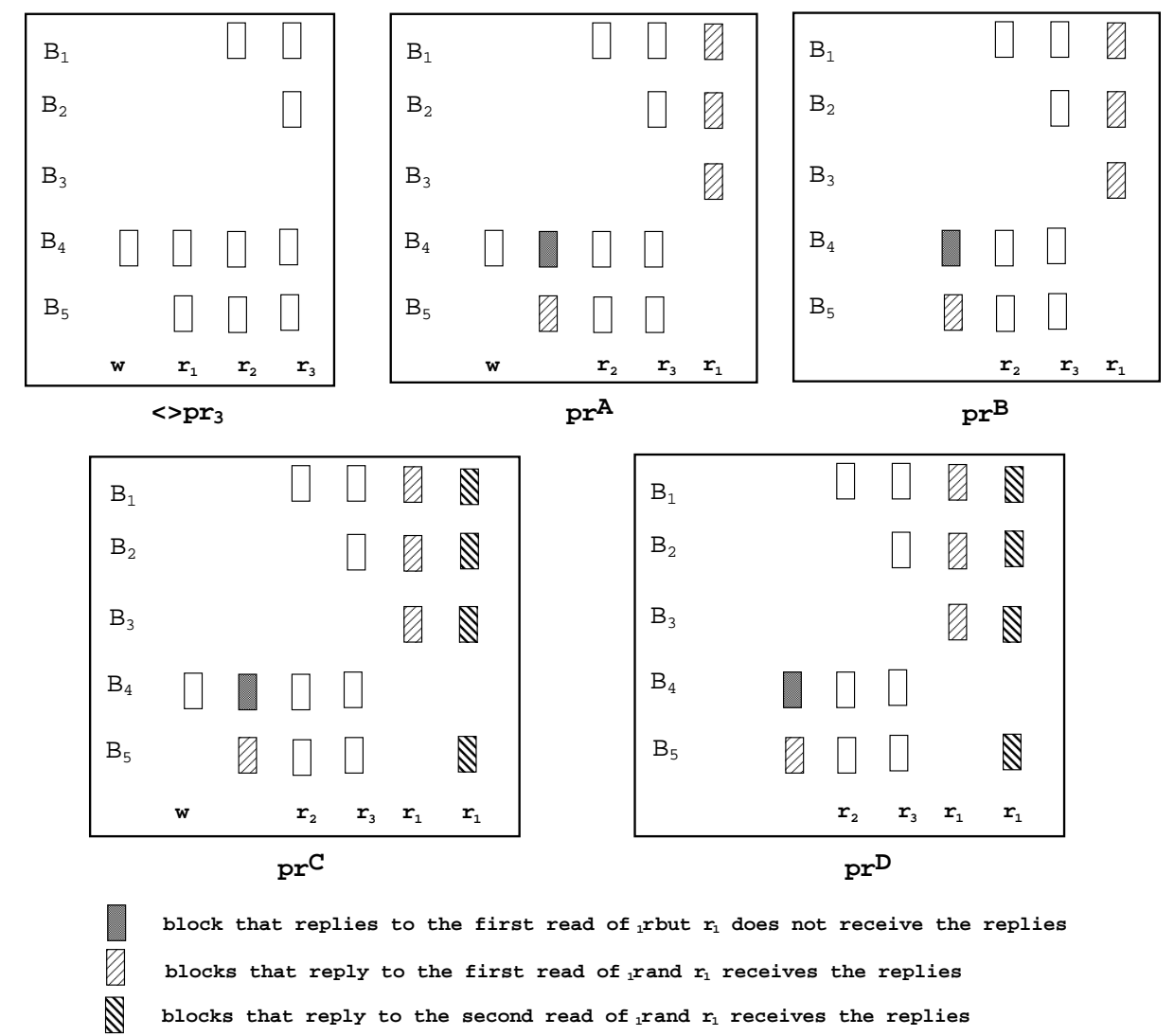

Fig. 4. Partial runs: $p r^{A}, p r^{B}, p r^{C}$ and $p r^{D}$

both runs. Thus $r_{1}$ 's read returns 1 in $\triangle p r_{1}$.

Starting from $\triangle p r_{1}$, we iteratively define the following partial runs for $2 \leq i \leq R$. Partial run $p r_{i}$ extends $\triangle p r_{i-1}$ by appending a complete read by $r_{i}$ that skips $B_{i}$. Partial run $\triangle p r_{i}$ is constructed by deleting from $p r_{i}$, all steps of the servers in block $B_{i}$. Since the last read in $p r_{i}$ by reader $r_{i}$ skips block $B_{i}, r_{i}$ cannot distinguish $p r_{i}$ from $\triangle p r_{i}$. More precisely, partial run $\triangle p r_{i}$ extends $w r_{i+1}$ by appending the following $i$ reads one after the other: ${ }^{6}$ for $1 \leq h \leq i, r_{h}$ does a read that skips $\left\{B_{j} \mid h \leq j \leq i\right\}$. Figure 3 depicts block diagrams of $p r_{i}$ and $\triangle p r_{i}$ with $R=3$. (The deletion of steps to obtain $\triangle p r_{i}$ from $p r_{i}$ is shown by crossing out the rectangles corresponding to the deleted steps.)

Reader $r_{1}$ 's read in $\triangle p r_{1}$ returns 1 . Since $p r_{2}$ extends $\triangle p r_{1}$, by atomicity, $r_{2}$ 's read in $p r_{2}$ returns 1 . However, as $r_{2}$ cannot distinguish $p r_{2}$ from $\triangle p r_{2}, r_{2}$ 's read in $\triangle p r_{2}$ returns 1. In general, since $p r_{i}$ extends $\triangle p r_{i-1}$, and $r_{i}$ cannot distinguish $p r_{i}$ from $\triangle p r_{i}$ (for all $i$ such that $2 \leq i \leq R$ ), it follows from a trivial induction that $r_{i}$ 's read in $\triangle p r_{i}$ returns 1 . In particular, $r_{R}$ reads 1 in $\triangle p r_{R}$.

${ }^{6}$ The first $i-1$ reads are incomplete whereas the last one is complete. 
Partial run $p r^{A}$. Consider the partial run $\triangle p r_{R}: w r_{R+1}$ extended by appending $R$ reads by each reader $r_{h}(1 \leq h \leq R)$ such that $r_{h}$ 's read skips $\left\{B_{j} \mid h \leq j \leq R\right\}$. The read by $r_{1}$ is incomplete in $\triangle p r_{R}$ : only servers in $B_{R+1}$ and $B_{R+2}$ send replies to $r_{1}$, and those reply messages are in transit. Observe that, in $\triangle p r_{R}$, only the servers in $B_{R+1}$ receive the WRITE message from the write(1) invocation. Consider the following partial run $p r^{A}$ which extends $\triangle p r_{R}$ as follows. After $\triangle p r_{R}$, (1) $r_{1}$ receives the replies of its READ messages from $B_{R+2}$ (that were in transit in $\triangle p r_{R}$ ), (2) the servers in $B_{1}$ to $B_{R}$ receive the READ message from $r_{1}$ (that were in transit in $\triangle p r_{R}$ ) and reply to $r_{1},(3)$ reader $r_{1}$ receives these replies from servers in $B_{1}$ to $B_{R}$, and then $r_{1}$ returns from the read invocation. (Notice that, $r_{1}$ received replies from $R+1$ blocks, and so, must return from the read.) However, $r_{1}$ does not receive the replies from servers in $B_{R+1}$ (that were in transit in $\triangle p r_{R}$ ). Figure 4 depicts block diagrams for $p r^{A}$ with $R=3$.

Partial run $p r^{B}$. Consider another partial run $p r^{B}$ with the same communication pattern as $p r^{A}$, except that write(1) is not invoked at all, and hence, servers in $B_{R+1}$ do not receive any WRITE message (Figure 4). Clearly, only servers in $B_{R+1}$, the writer, and the readers $r_{2}$ to $r_{R}$ can distinguish $p r^{A}$ from $p r^{B}$. Reader $r_{1}$ cannot distinguish the two partial runs because it does not receive any message from the servers in $B_{R+1}$, the writer, or other readers. By atomicity, $r_{1}$ 's read returns (the initial value of the register) $\perp$ in $p r^{B}$ because there is no write $(*)$ invocation in $p r^{B}$, and hence, $r_{1}$ 's read returns $\perp$ in $p r^{A}$ as well.

Partial runs $p r^{C}$ and $p r^{D}$. Notice that, in $p r^{A}$, even though $r_{1}$ 's read returns $\perp$ after $r_{R}$ 's read returns $1, p r^{A}$ does not violate atomicity, because the two reads are concurrent. We construct two more partial runs: (1) $p r^{C}$ is constructed by extending $p r^{A}$ with another complete read by $r_{1}$, which skips $B_{R+1}$, and (2) $p r^{D}$ is constructed by extending $p r^{B}$ with another complete read by $r_{1}$, which skips $B_{R+1}$ (Figure 4). Since $r_{1}$ cannot distinguish $p r^{A}$ from $p r^{B}$, and $r_{1}$ 's second read skips $B_{R+1}$ (i.e., the servers which can distinguish $p r^{A}$ from $p r^{B}$ ), it follows that $r_{1}$ cannot distinguish $p r^{C}$ from $p r^{D}$ as well. Since there is no write(*) invocation in $p r^{D}, r_{1}$ 's second read returns $\perp$ in $p r^{D}$, and hence, $r_{1}$ 's second read in $p r^{C}$ returns $\perp$. Since $p r^{C}$ is an extension of $p r^{A}, r_{R}$ 's read in $p r^{C}$ returns 1 . Thus, in $p r^{C}, r_{1}$ 's second read returns $\perp$ and succeeds $r_{R}$ 's read which returns 1 . Clearly, partial run $p r^{C}$ violates atomicity.

\section{ARBITRARY FAILURE MODEL}

An arbitrary failure can either correspond to a crash or a malicious behavior. A process is malicious if it deviates from the algorithm assigned to it in a way that is different from simply stopping all activities (crashing). We distinguish two resilience thresholds: $b$ and $t$ [Lamport 2003]. Just as in the crash-stop model, a maximum number of $t$ processes can crash. However, out of these $t$ processes, up to $b$ processes can be malicious. We therefore always have $b \leq t$.

In the literature, the special case where $b=t$ is usually considered. There are several advantages of distinguishing these two thresholds. First, we highlight the influence on the lower bounds of (1) the malicious behavior of the processes and (2) 


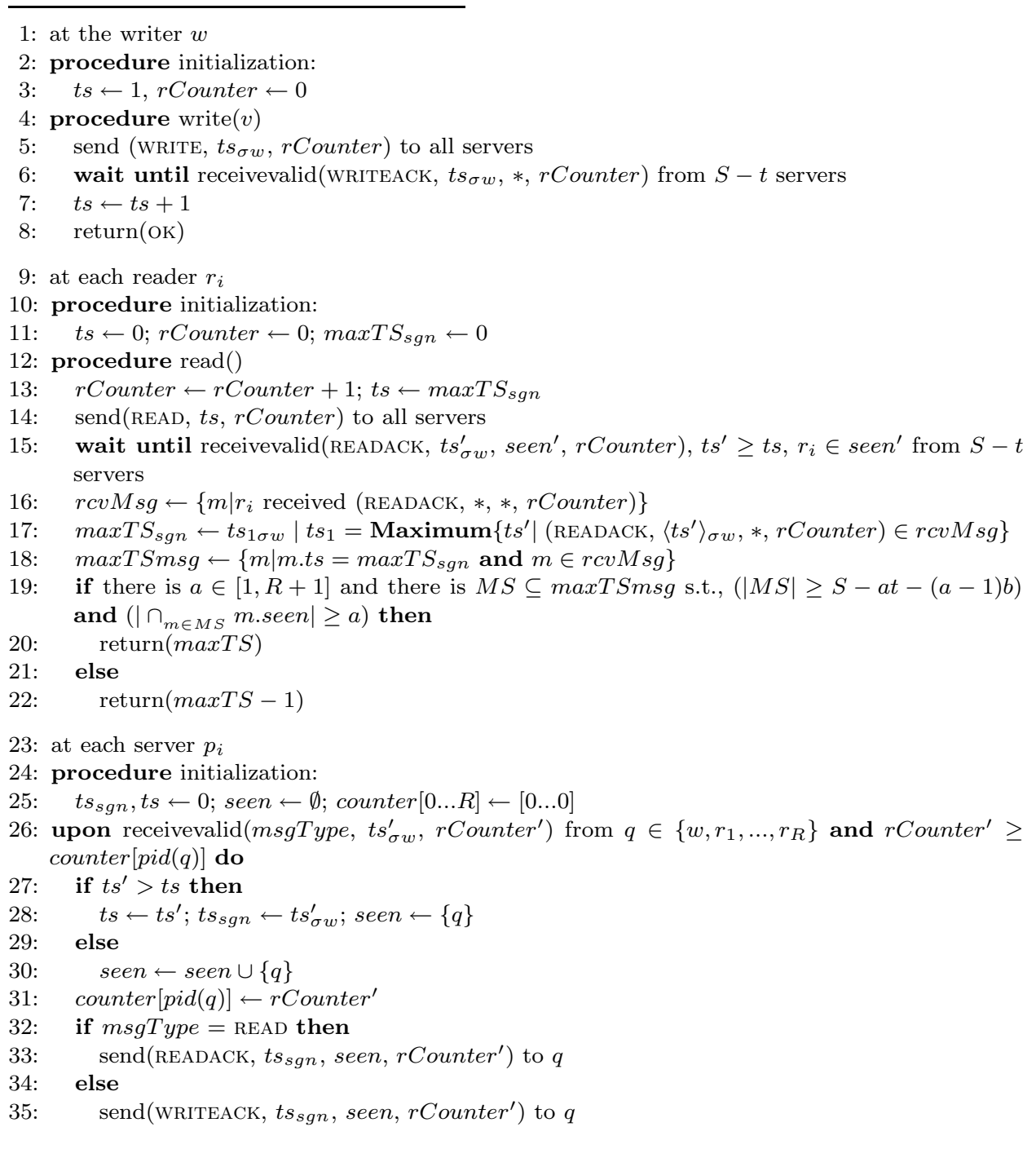

Fig. 5. Fast SWMR atomic register implementation with $S>(R+2) t+(R+1) b$

the processes' non-responsiveness. In the boundary case, where $b=0$, the failure model becomes non-arbitrary, i.e., the traditional crash failure model. Hence, the bounds derived in this paper for the general case with parameters $b$ and $t$ bridge the gap between arbitrary and crash failure models, by establishing a result that is applicable to both.

\subsection{A Fast Implementation}

We describe in this section a fast implementation in the arbitrary failure model assuming $S>(R+2) t+(R+1) b$ which is equivalent to $R<\frac{S+b}{t+b}-2$ (Figure 5 ). 
The algorithm is similar to the one presented in Section 4 except for a few key differences. First of all, the writer digitally signs each value [Rivest et al. 1978]. Digital signatures allow us to make the following assumptions:

Property 1. Authentication: readers can check that a value returned by a server was in fact written by the writer.

PROPERTY 2. Unforgeability: it is impossible to forge the digital signature of the writer.

Apart from the addition of digital signatures, the write mechanism is unchanged and the writer waits for the response of $S-t$ servers.

Our read procedure begins with servers issuing a READ message containing the highest signed timestamp encountered in the previous read invocation (lines 13-14). In a way, the reader writes back this timestamp, signed by the writer $\left(t s_{\sigma w}\right)$, to all servers. During the first read invocation, the reader issues a read message with the default timestamp 0 , which is also the initial timestamp at servers and writer. We assume that this initial value is not digitally signed by the writer. Then, the reader collects responses from $S-t$ servers containing the latest timestamps signed by the writer encountered by the servers (including the one being written back by the reader). The reader then selects the highest timestamp, denoted by $\max T S_{\text {sgn }}$ in Figure 2 (that will be written back by the reader in its next read invocation).

The mechanism of the read procedure is also very similar, except for the predicate which checks if the latest value has been seen by a sufficient number of processes. Consider the case of a write with timestamp $t s$ that is followed by a read. In the first partial run $p r_{1}$, the write completes by writing $t s$ at $S-t$ servers, out of which at least $S-t-b$ are non-malicious, let this set of servers be $S_{1}$. Subsequently, a reader reads from a set $S_{2}$ (of $S-t$ servers) that overlaps at $S-2 t-b$ (non-malicious) servers with $S_{1}$, i.e., misses $t$ servers in $S_{1}$. By atomicity, the read returns $t s$. In the second partial run $p r_{2}$, with a failure pattern different from $p r_{1}$, the write is incomplete and the writer writes $t s$ only to $S-2 t-b$ servers (possibly malicious) in $S 1 \cap S 2$. A subsequent reader that reads from $S_{2}$ cannot distinguish $p r_{1}$ from $p r_{2}$, and returns $t s$. If we extend each partial run with another read by a distinct reader that misses $t$ servers from $S 1 \cap S 2$, and accounting for the possibility that another $b$ servers are malicious, it is easy to see that the new read has to return $t s$, even if it sees $t s$ at $S-3 t-2 b$ servers that have already replied to both the write and the first read. Thus the predicate for the read procedure is as follows: if there is $a \geq 1$ such that the reader receives $\max T S_{\text {sgn }}$ in at least $S-a t-(a-1) b$ messages, and there are at least $a$ processes that are in the list seen of each of these $S-a t-(a-1) b$ messages, then the predicate is true.

We now prove the correctness of the fast implementation depicted in Figure 5. We do not assume that the lines in Figure 5 are atomic: processes may crash in the middle of a line or in between two lines. In particular, while sending messages to a set of processes, the sending process may crash after sending messages to an arbitrary subset. We assume that, if a process receives an incomplete message, the process can detect that the message is incomplete, and ignores such a message. 
It is obvious that read and write procedures complete in one round-trip. To show atomicity, we recall that the write procedure directly writes the timestamp. Thus the conditions in Section 3.1 reduce to the following: (1) if a read returns, it returns a non-negative integer, (2) if a read $r d$ is complete and it succeeds some write $(k)$, then $r d$ returns $l$ such that $l \geq k$, (3) if a read $r d$ returns $k(k \geq 1)$, then write $(k)$ either precedes $r d$ or is concurrent to $r d$, and (4) if some read $r d 1$ returns $k(k \geq 0)$ and a read $r d 2$ that succeeds $r d 1$ returns $l$, then $l \geq k$. The proofs of the first condition is trivial and it is not difficult to see that the third property holds, having in mind the unforgeability property of the writer's digital signature. Below, we show the other two. In the proofs we refer to the global clock; however processes do not access this global clock.

LEMMA 6. If a non-malicious server $s$ sets ts to $x$ at time $T$, then $s$ never sets ts to a value that is lower than $x$ after time $T$.

PROOF: obvious from line 33 .

LEMMA 7. If a read() sends READ messages with $t s=x$, then the read does not return a value smaller than $x$.

PROOF: recall that a read should return a value only if at most $t$ servers are faulty (liveness). Suppose read $r d$ by $r_{i}$ sends a READ message with $t s=x$. From line 33, every READACK message received by $r d$ from a non-malicious server is with $t s \geq x$. Reader awaits for $S-t$ READACK messages before returning a value. Moreover, reader discards all READACK messages that have a timestamp less than $x$, as those READACK messages are clearly from malicious servers. Eventually, if there are at most $t$ server failures, $r d$ receives READACK messages from $S-t$ non-malicious servers. Let $z$ be the maximum of those timestamps (i.e., $\max T S_{\text {sgn }}$ computed in line 20). Clearly, $z \geq x$. Notice that $r d$ returns either $z$ or $z-1$. There are the following two cases to consider. (1) If $z>x$, then clearly, the return value is not smaller than $x$. (2) If $z=x$, then every READACK message received by $r d$ has $t s=x$ (as all READACK that are received, and not discarded, are from nonmalicious servers) and has $r_{i} \in$ seen. Since $r d$ receives $S-t$ READACK messages from non-malicious servers, the predicate in line 22 of $r d$ holds with $a=1$. Hence, rd returns $x$.

LEMMA 8. If a read $r d$ is complete and it succeeds some write $(k)$, then $r d$ returns $l$ such that $l \geq k$.

PRoOF: suppose that write $w r$ (by $w$ ) writes $k$ and precedes read $r d$ (by reader $\left.r_{j}\right)$. Let $S 1$ be the set of $S-t$ servers from which $w r$ received WRITEACK messages in line 7 , and let $S 2$ be the set of $S-t$ servers from which $r d$ received READACK messages in line 18. Let $S 12=S 1 \cap S 2$ and $S 12_{n m}$ a subset of $S 12$ that contains only non-malicious servers. Obviously, $\left|S 12_{n m}\right| \geq S-2 t-b$. Let $z$ be the maximum timestamp received by $r d$ from servers in $S 2$. Observe that $r d$ returns either $z$ or $z-1$. 
When a non-malicious server in $S 1$ replies to a WRITE message from $w r$, its $t s$ is $k$. The server's $t s$ is not higher than $k$ because, unless the writer receives WRITEACK from all servers in $S 1$, it does not complete write $(k)$, and hence, no timestamp higher than $k$ is present in the system until all servers in $S 1$ reply to write $(k)$. From Lemma 6, non-malicious servers in $S 1$ (and hence, in $S 12_{n m}$ ) reply $t s \geq k$ to $r d$ because write $(k)$ precedes $r d)$. Thus, the highest timestamp received by $r d$, $z \geq k$. There are the following two cases to consider:

$-z>k$

Since $r d$ returns either $z$ or $z-1$, it follows that $r d$ does not return a timestamp lower than $k$.

$-z=k$

We know that every (non-malicious) server in $S 12_{n m}$ replies to $r d$ with $t s \geq k$, and $z=k$ is the maximum timestamp received by $r d$ from servers in $S 2 \supseteq$ $S 12 \supseteq S 12_{n m}$. Thus every server in $S 12_{n m}$ replies $t s=k$ to $r d$. Let $M S$ be the set of READACK messages sent by servers in $S 12_{n m}$ to $r d$. Since every server in $S 12_{n m}$ replies $t s=k$ to $w r$ before sending $t s=k$ to $r d$, for every message $m$ in $M S, w \in$ m.seen. Furthermore, from line $36, r_{j} \in$ m.seen. Thus, $\left\{w, r_{j}\right\} \subseteq \cap_{m \in M S}$ m.seen. As $\left|S 12_{n m}\right| \geq S-2 t-b$, in $r d$, the predicate in line 22 holds with $a=2$. Consequently, rd returns $z=k$.

LEMMA 9. If some read $r d 1$ returns $x(x \geq 0)$ and a read $r d 2$ that succeeds $r d 1$

returns $y$, then $y \geq x$.

PROOF: suppose that read $r d 1$ by process $r_{j}$ returns $x$, read $r d 2$ by process $r_{k}$ returns $z$, and $r d 1$ precedes $r d 2$. Suppose $r_{j}=r_{k}$. Then, in the read immediately after $r d 1, r_{j}$ sends a READ message with $t s \geq x$, and hence, from Lemma 7 , the read returns a value greater than or equal to $x$. Using Lemma 7 and a simple induction, we can derive that any read by $r_{j}$ which follows $r d 1$ (including $r d 2$ ) returns $t s \geq x$. So in the rest of the proof we assume that $r_{j} \neq r_{k}$.

Let $S 1$ and $S 2$ be the set of servers (of size $S-t$ ) from which reads $r d 1$ and $r d 2$, respectively, receive $S-t$ READACK messages in line 18. Let $T S 1$ be the highest timestamp received by $r d 1$ from processes in $S 1$ (i.e., the $\max T S_{\text {sgn }}$ evaluated in line 20of $r d 1$ ). Similarly, let $T S 2$ be the highest timestamp received by $r d 2$ from the processes in $S 2$. There are the following two cases to consider:

$\langle 1\rangle$. the predicate in line 20 does not hold in $r d 1$.

It follows that $x=T S 1-1$. Thus some servers have sent $t s=T S 1=x+1$ to $r d 1$, and hence, write $(x+1)$ has started before $r d 1$ is completed. Thus write $(x)$ has completed before $r d 1$ is completed. Since $r d 1$ precedes $r d 2$, it follows that write $(x)$ precedes $r d 2$. From Lemma $8, r d 2$ returns $y \geq x$.

$\langle 1\rangle 2$. the predicate in line 20 holds in $r d 1$.

It follows that $x=T S 1$, and there is some $a \in[1, R+1]$ such that there is a set $M S$ consisting of at least $S-a t-(a-1) b$ messages received by $r d 1$ with $t s=x$ and $\mid \cap_{m \in M S}$ m.seen $\mid \geq a$. Let $S 12 \subseteq S 1$ be the set of servers which sent the messages that are in $M S$. Since $a \in[1, R+1]$ and $S>(R+2) t+(R+1) b$, $|S 12|=|M S|=S-a t-(a-1) b>t+b$. There are the following two cases to 
consider:

$\langle 2\rangle$ 1. $y=T S 2$

$y=T S 2$. Since, $|S 12|>t+b$ and $|S 2|=S-t$, there is a non-malicious server $p_{i} \in S 2 \cap S 12$. Since $r d 1$ precedes $r d 2, p_{i}$ first replies $t s=x$ to $r d 1$ then replies to $r d 2$. From Lemma 6 , it follows that $p_{i}$ replies to $r d 2$ with $t s \geq x$. Thus the highest $t s$ in $S 2$ (i.e., $T S 2=y$ ) is greater than or equal to $x$.

$\langle 2\rangle 2 . y=T S 2-1$

There are the following two subcases to consider:

$\langle 3\rangle$ 1. $y+1 \neq x$

As in case $\langle 2\rangle 1$, we can show that there is a non-malicious server $p_{i} \in$ $S 2 \cap S 12$, and $p_{i}$ replies to $r d 2$ with $t s \geq x$. Thus the highest $t s$ in $S 2$ (i.e., $T S=y+1)$ is greater than or equal to $x$. Since $y+1 \neq x$, it follows that $y+1>x$, and hence, $y \geq x$.

$\langle 3\rangle 2 . y+1=x$

Consider the set of servers $S 2 \cap S 12$. As $|S 12|=S-a t-(a-1) b$ and $|S 2|=S-t$, so $|S 2 \cap S 12 \cap S n m| \geq S-(a+1) t-a b \geq 1$, where $S n m$ is a set of non-malicious servers $(|S n m| \geq S-b)$. Since $r d 1$ precedes $r d 2$ and non-malicious processes in $S 12$ replies $t s=x$ to $r d 1$, non-malicious processes in $S 2 \cap S 12$ reply to $r d 2$ with $t s \geq x$. Since $y+1$ is the maximum $t s$ in $S 2$, every non-malicious process in $S 2 \cap S 12$ replies to $r d 2$ with $t s=x=y+1$. There are the following two cases to consider:

$\langle 4\rangle 1 . a \leq R$

Then $|S 2 \cap S 12 \cap S n m| \geq S-(a+1) t-a b>t+b$. Let $M S 1$ be the set of READACK messages from processes in $S 2 \cap S 12 \cap S n m$ (nonmalicious processes from $S 1 \cap S 12$ ) to $r d 1$. From the definition of $M S 1$ and $M S, M S 1 \subseteq M S .^{7}$ Thus, $\cap_{m \in M S 1}$ m.seen $\supseteq \cap_{m \in M S}$ m.seen. Thus, $\mid \cap_{m \in M S 1}$ m.seen $\mid \geq a$. There are two cases to consider:

$\langle 5\rangle 1 . r_{k} \notin \cap_{m \in M S 1}$ m.seen

Let $M S 2$ be the set of messages received by $r d 2$ from processes in $S 2 \cap$ $S 12 \cap S n m$. For any (non-malicious) server $p_{i} \in S 2 \cap S 12 \cap S n m$, let $m 1_{i}$ and $m 2_{i}$ be the messages sent by $p_{i}$ in $M S 1$ and $M S 2$ respectively. We know that $m 1_{i}$.ts $=m 2_{i}$.ts $=x$. Since $m 1_{i}$ is sent before $m 2_{i}$ and the $t s$ is the same in both messages, $m 1_{i}$.seen $\subseteq m 2_{i}$.seen. Thus $\cap_{m \in M S 1}$ m.seen $\subseteq \cap_{m \in M S 2}$ m.seen. Since every process which replies to $r d 2$, first adds $r_{k}$ to its seen set, $r_{k} \in \cap_{m \in M S 2}$ m.seen. Since $r_{k} \notin$ $\cap_{m \in M S 1}$ m.seen, it follows that $\mid \cap_{m \in M S 2}$ m.seen $|\geq| \cap_{m \in M S 1}$ m.seen $\mid+1 \geq$ $a+1$. Since $|S 2 \cap S 12 \cap S n m| \geq S-(a+1) t-a b$, the number of message in $M S 2$ is at least $S-(a+1) t-a b$. As $a+1 \leq R+1$, the predicate in line 22 in $r d 2$ holds with $a+1$. Thus, the timestamp returned by $r d 2$ is $x=y+1$, a contradiction.

$\langle 5\rangle 2 . r_{k} \in \cap_{m \in M S 1}$ m.seen

Thus each server $p_{i}$ in $S 2 \cap S 12 \cap S n m$ has sent at least one READACK message with $t s=x$ to $r_{k}$, before $p_{i}$ sent the $M S 1$ message to $r_{j}$. Since the messages in $M S 1$ are sent before the completion of $r d 1$ (and

$\overline{{ }^{7} \text { See case }\langle 1\rangle} 2$ for the definition of $M S$. 
hence, before the invocation of $r d 2$ ), $r_{k}$ has invoked at least one read before $r d 2$. Let $r d 2 a$ be the last read of $r_{k}$ which precedes $r d 2$. Since $|S 2 \cap S 12 \cap S n m| \geq S-(a+1) t-a b>t+b$, there is at least one process $p_{i}$ in $S 2 \cap S 12 \cap S n m$ whose READACK message is received by $r d 2 a$, say message $m$. Now consider the last READACK message sent by $p_{i}$ to $r_{k}$ before $r d 2$ is invoked, say message $m^{\prime}$. Since we know that $p_{i}$ sent a READACK message with $t s=x$ to $r_{k}$ before sending a $M S 1$ message (which was in turn sent before $r d 2$ was invoked), from Lemma 6 it follows that $m^{\prime}$ was sent with $t s \geq x$. We now claim that $m=m^{\prime}$. By definition of $m^{\prime}$, either $m=m^{\prime}$ or $m^{\prime}$ is sent after $m$. Observe that $p_{i}$ checks counter $[k]$ before replying to $r_{k}$. Thus, once $m$ is sent by $p_{i}$, counter $[k]$ at $p_{i}$ is set such that $p_{i}$ can only reply to those message of $r_{k}$ which are sent from $r d 2 a$ or a subsequent read of $r_{k}$. Thus, if $m^{\prime}$ is sent after $m$, then $m^{\prime}$ is sent in response to $r d 2 a$, or $r d 2$, or a subsequent read of $r_{k}$. This contradicts the assumption that $p_{i}$ replies only once to $r d 2 a$ (because channels do not duplicate messages) and $m^{\prime}$ is sent before $r d 2$ is invoked. Thus $r d 2 a$ receives $m=m^{\prime}$. We have already shown that $m^{\prime}$ is sent with $t s \geq x$. Hence the highest $t s$ received by $r d 2 a$ is greater than or equal to $x$. It follows that $r d 2$ sends READ messages with $t s \geq x$. From Lemma $7, r d 2$ returns a timestamp greater than or equal to $x$. As $x=y+1, r d 2$ does not return $y$, a contradiction.

$\langle 4\rangle 2 . \quad a=R+1$

Since $\left|\left\{w, r_{1}, \ldots, r_{R}\right\}\right|=R+1$ and $\mid \cap_{m \in M S}$ m.seen $\mid \geq a=R+1$, we have $r_{k} \in \cap_{m \in M S}$ m.seen. Observe that $|S 12| \geq S-a t-(a-1) b>t+b$. (Recall that $S 12$ is the set of processes which sent the messages that are in $M S$.) Substituting $M S 1$ by $M S$, and $S 2 \cap S 12$ by $S 12$, in the argument for the previous case (case $5\langle 2\rangle$ ), we can show that $r d 2$ returns a value greater than or equal to $x$, a contradiction.

\subsection{Optimality}

The following proposition states that the resilience required by our fast implementation is indeed necessary.

Proposition 10. Let $t \geq 1, b \geq 0$ and $R \geq 2$. If $(R+2) t+(R+1) b \geq S$, then there is no fast atomic register implementation.

This proof is similar to the one in Section 5: we suppose by contradiction that $(R+2) t+(R+1) b \geq S$ and that there is a fast implementation $I$ of an atomic register (even with public key cryptography). We construct a partial run of the fast implementation $I$ that violates atomicity: a partial run in which some read returns 1 and a subsequent read returns an older value, namely, the initial value of the register, $\perp$. This run is different from the one in the previous proof.

Partial writes. Note that we assume that writer writes digitally signed information to servers, and that arbitrarily faulty servers cannot forge the writers signature. Consider a partial run $w r$ in which $w$ completes write(1) on the register. The invo- 
cation skips $T_{R+2}$. We define a series of partial runs each of which can be extended to $w r$. Let $w r_{R+2}$ be the partial run in which $w$ has invoked the write and has sent the WRITE message to all processes, and all WRITE messages are in transit. For $1 \leq i \leq R+1$, we define $w r_{i}$ as the partial run which contains an incomplete write(1) invocation that skips $\left\{T_{R+2}\right\} \cup\left\{T_{j} \mid 1 \leq j \leq i-1\right\} \cup\left\{B_{j} \mid 1 \leq j \leq i-1\right\}$. We make the following simple observations: (1) for $1 \leq i \leq R, w r_{i}$ and $w r_{i+1}$ differ only at servers in $T_{i} \cup B_{i},(2) w r$ is an extension of $w r_{1}$, such that, in $w r$, $w$ receives the replies (that are in transit in $w r_{1}$ ) and returns from the write invocation, and hence, (3) $w r$ and $w r_{1}$ differ only at $w$.

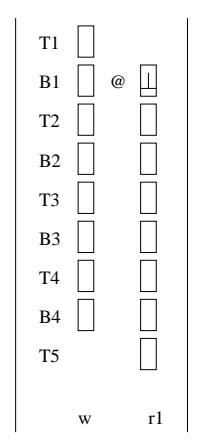

(a) $\operatorname{pr} 1$

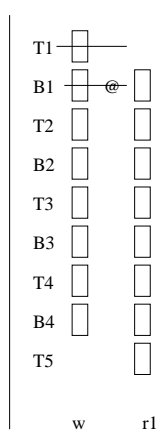

(b)

pr $1-\triangle p r 1$

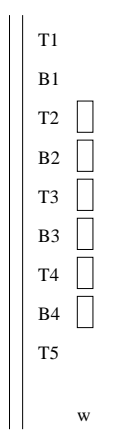

(c) $\triangle p r 1-p r 2$

(d) $p r 2-\triangle p r 2$

(e) $\triangle p r 2-p r 3$

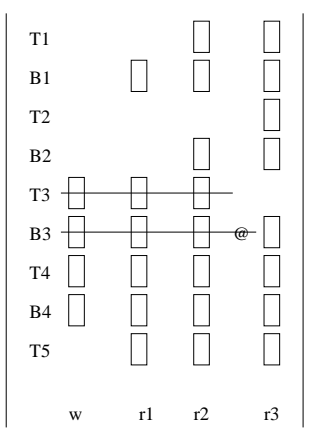

(f) $p r 3-\triangle p r 3$

Fig. 6. Partial runs $p r_{i}$ and $\triangle p r_{i}$

Block diagrams. We illustrate a particular instance of the proof in Figure 6 and Figure 4 (unchanged from Section 5), where $R=3$ and the set of servers are partitioned into nine blocks, $T_{1}$ to $T_{5}$ and $B_{1}$ to $B_{4}$. We depict an invocation inv through a set of rectangles, (generally) arranged in a single column. In the column corresponding to some invocation $i n v$, we draw a rectangle in the $i^{\text {th }}$ row, if all servers in block $B_{i}$ have received the READ or WRITE message from inv and have sent reply messages, i.e., we draw a rectangle in the $i^{t h}$ row if $i n v$ does not skip $B_{i}$. In partial run $p r_{i}$, we denote the failure of $B_{i}$ by @ ( $B_{i}$ "looses its memory"). 
Appending reads. Partial run $p r_{1}$ extends $w r$ by having block $B_{1}$ failing arbitrarily upon completion of write(1) and appending a complete read by $r_{1}$ that skips block $T_{1} . B_{1}$ fails in such a way that it behaves as if it never any message (i.e., a message from invocation write(1)). We say that $B_{1}$ fails and loses its memory. Observe that $r_{1}$ cannot distinguish $p r_{1}$ from some partial run $\triangle p r_{1}$, that extends $w r_{2}$ by appending a complete read by $r_{1}$ that skips $T_{1}$. To see why, notice that $w r$ and $w r_{2}$ differ at $w$ and at blocks $T_{1}$ and $B_{1}$, and $r_{1}$ does not receive any message from process $w$ and block $T_{1}$ in both runs and it received the same message from block $B_{1}$ in both runs. By liveness property, $r_{1}$ 's read in $\triangle p r_{1}$ must return some value $x$ (as it cannot wait for the completion of the writer's invocation, nor a message from $w)$. As $r_{1}$ cannot distinguish $\triangle p r_{1}$ from $p r_{1}$, it returns the same value $x$ in $p r_{1}$ as well, and by atomicity, in $p r_{1} x$ must equal 1 . Therefore, in $\triangle p r_{1} r_{1}$ also returns 1 . Starting from $\triangle p r_{1}$, we iteratively define the following partial runs for $2 \leq i \leq R$. Partial run $p r_{i}$ extends $\triangle p r_{i-1}$ by: (1) block $B_{i}$ failing arbitrarily in such a way that it behaves as if it never received any message (loses memory) and (2) appending a complete read by $r_{i}$ that skips $T_{i}$. Partial run $\triangle p r_{i}$ is constructed by deleting from $p r_{i}$, all steps of the servers in block $T_{i}$ and all steps of servers in block $B_{i}$ up to the instant in which $B_{i}$ lost its memory (including that particular step). Since the last read in $p r_{i}$ by reader $r_{i}$ skips block $T_{i}, r_{i}$ cannot distinguish $p r_{i}$ from $\triangle p r_{i}$, as in both runs $r_{i}$ receives the same messages from $B_{i}$. More precisely, partial run $\triangle p r_{i}$ extends $w r_{i+1}$ by appending the following $i$ reads one after the other: ${ }^{8}$ for $1 \leq h \leq i-1, r_{h}$ does a read that skips $\left\{T_{j} \mid h \leq j \leq i\right\} \cup\left\{B_{j} \mid h+1 \leq j \leq i\right\}$ and $r_{i}$ does a (complete) read that skips $T_{i}$. Figure 6 depicts block diagrams of $p r_{i}$ and $\triangle p r_{i}$ with $R=3$. (The deletion of steps to obtain $\triangle p r_{i}$ from $p r_{i}$ is shown by crossing out the rectangles corresponding to the deleted steps.)

Reader $r_{1}$ 's read in $\triangle p r_{1}$ returns 1. By liveness requirements in $\triangle p r_{2} r_{2}$ must return some value, say $x_{2}$. However, as $r_{2}$ cannot distinguish $p r_{2}$ from $\triangle p r_{2}$, so it must return a value $x_{2}$ in $p r_{2}$, as well. Since $p r_{2}$ extends $\triangle p r_{1}$, by atomicity, $r_{2}$ 's read in $p r_{2}$ must return $x_{2}=1$. Therefore, $r_{2}$ 's read in $\triangle p r_{2}$ returns 1 . In general, since $p r_{i}$ extends $\triangle p r_{i-1}$, and $r_{i}$ cannot distinguish $p r_{i}$ from $\triangle p r_{i}$ (for all $i$ such that $2 \leq i \leq R$ ), in which it must return a value, it follows from a trivial induction that $r_{i}$ 's read in $\triangle p r_{i}$ returns 1 . In particular, $r_{R}$ reads 1 in $\triangle p r_{R}$. Moreover, note that in $\triangle p r_{R}$ no server is faulty.

Partial run $p r^{A}$. Consider the partial run $\triangle p r_{R}$ : $w r_{R+1}$ extended by appending $R$ reads by each reader $r_{h}(1 \leq h \leq R-1)$ such that $r_{h}$ 's read skips $\left\{T_{j} \mid h \leq\right.$ $j \leq R-1\} \cup\left\{B_{j} \mid h+1 \leq j \leq R-1\right\}$ and a read by reader $r_{R}$ skips $T_{R}$ only. The read by $r_{1}$ is incomplete in $\triangle p r_{R}$ : only servers in $B_{1}, T_{R+1}, B_{R+1}$ and $T_{R+2}$ send replies to $r_{1}$, and those reply messages are in transit. Observe that, in $\triangle p r_{R}$, only the servers in $T_{R+1}$ and $B_{R+1}$ receive the WRITE message from the write(1) invocation. Consider the following partial run $p r^{A}$ which differs from the $\triangle p r_{R}$ in the following: (0) Upon reception of message from write(1) invocation, $B_{R+1}$ fails arbitrarily in such a way that, from that point on, it sends replies to all processes but $r_{1}$ as if it was not faulty, and to $r_{1}$ as if it never received a write(1) message.

${ }^{8}$ The first $i-1$ reads are incomplete whereas the last one is complete. 
Moreover, after completion of read by $r_{R}$, (1) $r_{1}$ receives the replies of its READ messages from $T_{R+2}$ and $B_{1}$ (that were in transit in $\triangle p r_{R}$ ) and $B_{R+1}$ (the faulty ones), (2) the servers in $T_{1}$ to $T_{R}$ and $B_{2}$ to $B_{R}$ receive the READ message from $r_{1}$ (that were in transit in $\triangle p r_{R}$ ) and reply to $r_{1},(3)$ reader $r_{1}$ receives these replies from servers in $T_{1}$ to $T_{R}$ and $B_{2}$ to $B_{R}$, and then $r_{1}$ returns from the read invocation. (Notice that, $r_{1}$ received replies from all blocks but $T_{R+1}$, and so, must return from the read. However, $r_{1}$ does not receive the replies from servers in $T_{R+1}$.

Partial run $p r^{B}$. Consider another partial run $p r^{B}$ with the same communication pattern as $p r^{A}$, except that write(1) is not invoked at all and block $B_{R+1}$ is not faulty. Hence, servers in $T_{R+1}$ do not receive any WRITE message (Figure 4 ). Clearly, only servers in $T_{R+1}, B_{R+1}$, the writer, and the readers $r_{2}$ to $r_{R}$ can distinguish $p r^{A}$ from $p r^{B}$. Reader $r_{1}$ cannot distinguish the two partial runs because it does not receive any message from the servers in $T_{R+1}$, the writer, or other readers and it receives the same message from the servers in $B_{R+1}$ in both runs. By atomicity, $r_{1}$ 's read returns (the initial value of the register) $\perp$ in $p r^{B}$ because there is no write $(*)$ invocation in $p r^{B}$, and hence, $r_{1}$ 's read returns $\perp$ in $p r^{A}$ as well.

Partial runs $p r^{C}$ and $p r^{D}$. Notice that, in $p r^{A}$, even though $r_{1}$ 's read returns $\perp$ after $r_{R}$ 's read returns $1, p r^{A}$ does not violate atomicity, because the two reads are concurrent. We construct two more partial runs: (1) $p r^{C}$ is constructed by extending $p r^{A}$ with another complete read by $r_{1}$, which skips $T_{R+1}$, and (2) $p r^{D}$ is constructed by extending $p r^{B}$ with another complete read by $r_{1}$, which skips $T_{R+1}$ (Figure 4). Since $r_{1}$ cannot distinguish $p r^{A}$ from $p r^{B}$, and $r_{1}$ 's second read skips $T_{R+1}$ (i.e., the servers which can distinguish $p r^{A}$ from $p r^{B}$ ), it follows that $r_{1}$ cannot distinguish $p r^{C}$ from $p r^{D}$ as well. Since there is no write $(*)$ invocation in $p r^{D}, r_{1}$ 's second read returns $\perp$ in $p r^{D}$, and hence, $r_{1}$ 's second read in $p r^{C}$ returns $\perp$. Since $p r^{C}$ is an extension of $p r^{A}, r_{R}$ 's read in $p r^{C}$ returns 1 . Thus, in $p r^{C}, r_{1}$ 's second read returns $\perp$ and succeeds $r_{R}$ 's read which returns 1 . Clearly, partial run $\mathrm{pr}^{\mathrm{C}}$ violates atomicity.

\section{MULTIPLE WRITERS}

The atomicity definition presented in Section 3 extends to multi-writer multi-reader (MWMR) registers as well. In the impossibility proof below, we use two simple properties of MWMR atomic register which can be easily deduced from atomicity. In any partial run (property $\mathbf{P 1}$ ) if a write $w r$ that writes $v$, precedes some read $r d$, and all other writes precede $w r$, then if $r d$ returns, it returns $v$, and (property P2) if there are two reads such that all writes precede both reads, then the reads do not return different values.

The proposition below states that there cannot exist a fast multi-writer atomic register implementation. The proof is written for the crash-stop model. But by extension the impossibility directly applies to the malicious failure model.

Proposition 11. Let $t \geq 1, W \geq 2, R \geq 2$. Any atomic register implementation has a run in which some complete read or write is not fast. 
Proof: it is sufficient to show the impossibility in a system where $W=R=2$, and $t=1$. Let the writers be $w_{1}$ and $w_{2}$, and the readers be $r_{1}$ and $r_{2}$. Let $s_{1}$ to $s_{S}$ be the servers. Suppose by contradiction that there is a fast implementation of an atomic register in this system. To show the desired contradiction, we construct a series of runs, each consisting of two writes followed by a read.

Since the writer, any number of readers, and up to $t$ servers might crash in our model, the invoking process can only wait for reply messages from $S-t$ servers. Given that we assume a fast implementation, on receiving a READ (or a WRITE) message, the servers cannot wait for messages from other processes, before replying to the READ (or the WRITE) message. We can thus construct partial runs of a fast implementation such that only READ (or WRITE) messages from the invoking processes to the servers, and the replies from servers to the invoking processes, are delivered in those partial runs. All other messages remain in transit. In particular, no server receives any message from other servers, and no invoking process receives any message from other invoking processes. In our proof, we only construct such partial runs.

We say that a complete invocation inv skips a server $s_{i}$ in a partial run if every server distinct from $s_{i}$ receives the READ or the WRITE message from $i n v$ and replies to that message, inv receives those replies and returns, and all other messages are in transit. In other words, only $s_{i}$ does not receive READ or WRITE message from inv. Since $t=1$, any complete invocation may skip at most one server. If a complete invocation does not skip any servers, we say that the invocation is skip-free.

Consider a partial run run $_{1}$ constructed with the following three non-overlapping invocations: (1) a skip-free write $(2)$ by $w_{2}$, that precedes $(2)$ a skip-free write(1) by $w_{1}$, that in turn precedes $(3)$ a skip-free $\operatorname{read}()$ by $r_{1}$. From property $\mathrm{P} 1$, the read returns 1 .

We now construct a similar partial run run $_{2}$ in which the order of the two writes are interchanged: (1) a skip-free write(1) by $w_{1}$, that precedes (2) a skip-free write(2) by $w_{2}$, that in turn precedes $(3)$ a skip-free $\operatorname{read}()$ by $r_{1}$. From property P1, the read returns 2 .

Consider a series of partial runs run $^{i}$, where $i$ varies from 1 to $S+1$. We define run $^{1}$ to be run $_{1}$. We iteratively define the remaining partial runs. We define run $^{i+1}$ to be identical to run $^{i}$ except in the following: $s_{i}$ receives the WRITE message (and replies to that message) from $w_{1}$ before the message from $w_{2}$ (i.e., the replies of $s_{i}$ are sent in the opposite order in $r^{i+1}$ from that in $\left.r u n^{1}\right)$. Since servers do not receive any message from other servers in the partial runs we construct, the only server that can distinguish run from $r u n^{i+1}$ is $s_{i}$. Also $w_{1}, w_{2}$ and $r_{1}$ can distinguish the two partial runs. It is easy to see that no server can distinguish run $^{S+1}$ from run $_{2}$, and hence, $r_{1}$ can not distinguish between the two runs as well. Thus $r_{1}$ returns 2 in $r n^{S+1}$, and run $^{S+1}$ and run $_{2}$ differ only at $w 1$ and $w 2$. Since $r 1$ returns 1 in $\operatorname{run}^{1}, 2$ in $r^{S+1}$, and either 1 or 2 in $\operatorname{run}^{i}(2 \leq i \leq S)$, there are two partial runs, run $^{i 1}$ and run $^{i 1+1}$, such that $1 \leq i 1 \leq S$ and the read by $r 1$ returns 1 in run $^{i 1}$ and returns 2 in run $^{i 1+1}$.

Consider a partial run run' which extends run $^{i 1}$ with a read by $r_{2}$ that skips $s_{i 1}$. From property P2, it follows that $r_{2}$ returns 1 . Similarly we construct a partial run run $^{\prime \prime}$ which extends run ${ }^{i+1}$ with a read by $r_{2}$ that skips $s_{i 1}$. Recall that, only $w_{1}$, 

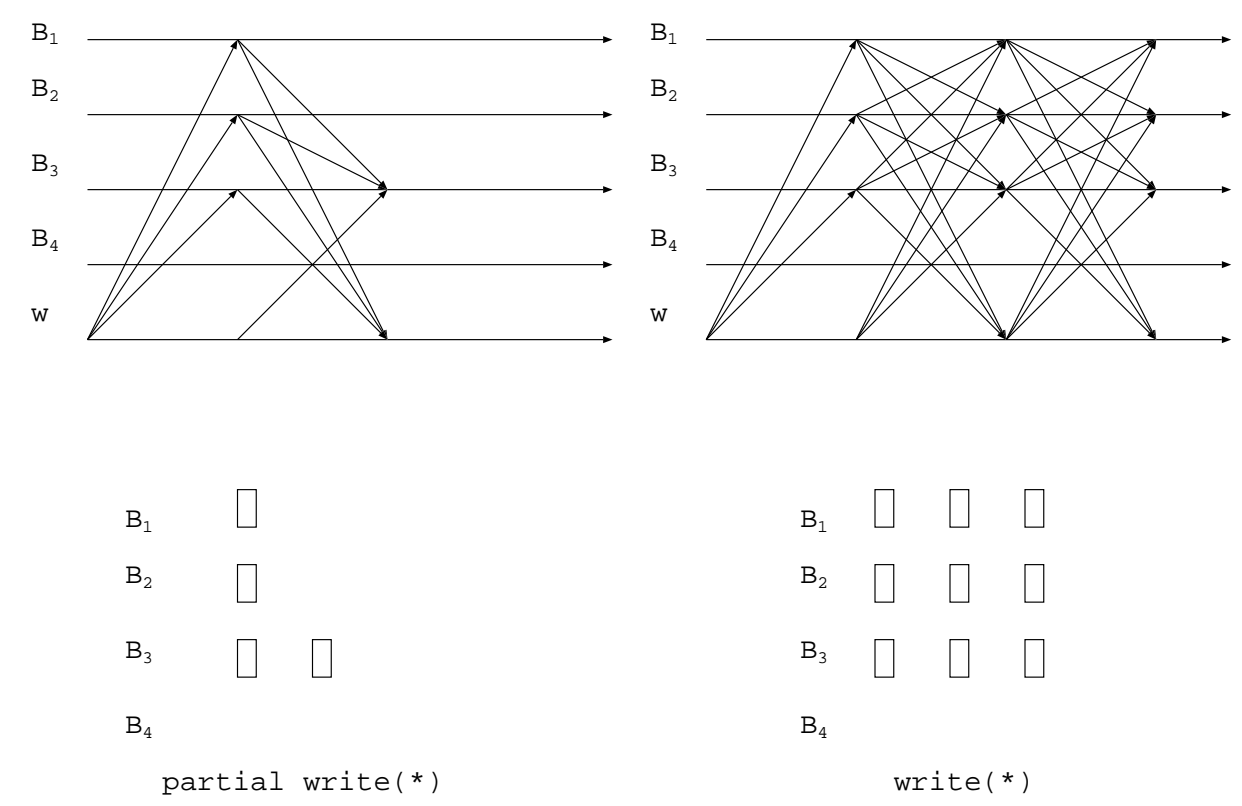

Fig. 7. Partial writes $(K=3, R=4)$

$w_{2}, r_{1}$ and $s_{i 1}$ can distinguish run $^{i 1}$ from $r u n^{i 1+1}$. Since $r_{2}$ skips $s_{i 1}$ in both run ${ }^{\prime}$ and run $^{\prime \prime}, r_{2}$ cannot distinguish the two partial runs. Thus $r_{2}$ returns 1 in run". However, $r_{1}$ returns 2 in run ${ }^{i 1+1}$, and hence, in returns 2 in run" as well. Clearly, run $^{i 1+1}$ violates property $\mathrm{P} 2$.

To see why the above proof does not apply to the single writer case, observe that in most partial runs in the above proof, the two writes are concurrent. However, in our system model, a process can invoke at most one invocation at a time. Thus we cannot construct partial runs with concurrent writes in the single-writer case.

\section{WHEN "ATOMIC READS MUST WRITE"}

Our results revisit, in a message passing context, the folklore theorem that "atomic reads must write", borrowed from the shared-memory context [Lamport 1986; Attiya and Welch 1998]. In particular, a result from [Attiya and Welch 1998] states that, to simulate a multi-reader atomic register from single-reader atomic registers, at least one of the readers must write into some single-reader register. Along the same lines, when implementing atomic registers over weaker regular ${ }^{9}$ ones [Lamport 1986], a process that reads a value $v$ also needs to write it, in order to make sure that no other process will subsequently read an older value $v^{\prime}$ : with a regular register, even if a value $v^{\prime}$ is written before a value $v, v$ might be read before $v^{\prime}$, which is impossible with an atomic register.

Recently, [Fan and Lynch 2003] has shown that, in a message-passing system,

${ }^{9} \mathrm{~A}$ regular register is like an atomic register except when there is concurrency: a a reader might not return the last value written. 
every atomic read must modify the state of at least $t$ servers, which might be interpreted as a need for a second communication round-trip. However, in such a system, any message received by a server can potentially modify the server's state. Hence, a read can modify at least $S-t>t$ servers (assuming a majority of correct servers) in one round-trip. In fact, processes (servers) are smarter than basic (regular or single-reader) registers and might intuitively do a lot in one communication round-trip.

Our results also draw a sharp line between the time-complexity of regular [Lamport 1978] and atomic register implementations. For instance, in the crash-only model, there is a fast implementation of an SWMR regular register if and only if $t<S / 2$, irrespective of the number of readers (as long as this number is finite). In this model, we show that a fast implementation of a SWMR atomic register exists if and only if $t<\frac{S}{R+2}$. However, since fast atomic registers have exactly the same time-complexity as regular registers they are clearly the most interesting option where only a few readers are necessary. In an application where a high number of readers (or writers) are required a trade-off needs to be made. A regular register will provide speed at the expense of consistency and an atomic register will provide better consistency guarantees at the expense of speed.

\section{SUMMARY}

This paper establishes the exact conditions required for a fast implementation of an atomic read-write data structure, also called a register.

In the case of multiple writers, we proved that a fast implementation is impossible even if only one server can fail, and it can only do by crashing.

In the case of a single-writer where $t$ out of $S$ servers can fail by crashing, the number of readers must be smaller than $S / t-2$. In the general arbitrary failure model, this number must be smaller than $(S+b) /(t+b)-2$ where up to $b$ out of $t$ servers can be malicious.

\section{REFERENCES}

Attiya, H., Bar-Noy, A., And Dolev, D. 1995. Sharing memory robustly in message-passing systems. Journal of the ACM 42, 1, 124-142.

Attiya, H. And Welch, J. 1998. Distributed Computing. Fundamentals, Simulations, and Advanced Topics. McGraw-Hill.

FAn, R. AND Lynch, N. 2003. Efficient replication of large data objects. In Proceedings of the 17th International Symposium on Distributed Computing (DISC-17).

Herlihy, M. 1991. Wait-free synchronization. ACM Transactions on Programming Languages and Systems 13, 1 (January), 124-149.

Herlihy, M. And Wing, J. 1990. Linearizability: a correctness condition for concurrent objects. ACM Transactions on Programming Languages and Systems 12, 3 (July), 463-492.

LAmport, L. 1978. Time, clocks and the ordering of events in a distributed system. Communications of the ACM 21, 7, 558-565. 
LAMPORT, L. 1986. On interprocess communication. Distributed computing 1, 1 (May), 77-101.

LAmport, L. 2003. Lower bounds for asynchronous consensus. Future Directions in Distributed Computing, 22-23.

Lynch, N. And Shvartsman, A. 1997. Robust emulation of shared memory using dynamic quorum-acknowledged broadcasts. In Proceedings of the 27th Annual International Symposium on Fault-Tolerant Computing (FTCS'97).

LynCh, N. A. 1996. Distributed Algorithms. Morgan-Kaufmann.

Rivest, R. L., Shamir, A., And Adleman, L. M. 1978. A method for obtaining digital signatures and public-key cryptosystems. Communications of the ACM 21, 2, 120-126. 